\title{
Design of set-point weighting-based dynamic integral sliding mode control with nonlinear full-order state observers for quadcopter UAVs
}

\author{
Ahmad Riyad Firdaus* \\ Department of Electrical Engineering, \\ Politeknik Negeri Batam, \\ Batam, Indonesia \\ E-mail: rifi@polibatam.ac.id \\ * Corresponding author \\ M. O. Tokhi \\ School of Engineering, \\ London South Bank University, \\ London, United Kingdom \\ E-mail: tokhim@lsbu.ac.uk
}

\begin{abstract}
This research is to develop set-point weighting-based dynamic integral sliding mode control with nonlinear full-order state observers to deal with nonlinear and underactuated coupled systems, and unforeseen circumstances of quadcopter UAVs system. A comparative assessment through numerical simulations of sliding mode-based nonlinear observer approaches and Kalman filter is presented. These include quasi method, interval type-2 fuzzy logic system, super-twisting algorithm, higher order sliding mode observer, and extended Kalman filter. Chattering, noise rejection, estimation error and time required to track true states are evaluated to demonstrate the performance of each observer. In addition, to assess the proposed controller performance, maximum overshoot, rise time, chattering, and steady-state error are evaluated in relation to the use of each observer.
\end{abstract}

Keywords: Nonlinear systems; underactuated and coupled systems; integral dynamic sliding mode control; set-point weighting function; nonlinear full-order state observers.

Biographical notes: Ahmad Riyad Firdaus received his $\mathrm{PhD}$ in Automatic Control and Systems Engineering at the University of Sheffield, UK. He is a Lecturer at the Department of Electrical Engineering, Politeknik Negeri Batam, Indonesia. His research interests include issues related to nonlinear control systems, soft-computing, unmanned aerial vehicles (UAVs), and robotics. He is author of research papers published at national and international journals, and conference proceedings.

M. Osman Tokhi obtained his BSc (Electrical Engineering) from Kabul University (Afghanistan) in 1978 and $\mathrm{PhD}$ from Heriot-Watt University (UK) in 1988. He is a Chartered Engineer, Fellow of IET (Institution of Engineering and Technology), Senior Member of IEEE (Institute of Electronic and Electrical Engineering), and Member of IIAV (International Institute of Acoustics and Vibration) and of CLAWAR (Climbing and Walking Robots) Association. He has worked in various academic positions and in the industry. He is currently a professor in the School of Engineering, London South Bank University (UK). His main research interests include active control of noise and vibration, adaptive/intelligent control, assistive robotics, soft computing techniques for modelling and control of dynamic systems, and high-performance real-time computing. He has published extensively and has executed numerous research projects to successful completion in these areas.

\section{Introduction}

Various controllers have been developed for performance enhancement of quadcopter UAVs. Examples include proportional integral derivative (PID) control (Guo et al., 2017); linear quadratic regulator (LQR) (Jasim and $\mathrm{Gu}, 2019)$; model predictive control (MPC)
(Zanelli et al., 2017); and sliding mode control (SMC) (Zheng et al., 2014). Among these, The SMC method outperforms others in terms of dealing with nonlinearity, underactuation, and uncertainty. This is because the controller is designed to be insensitive to such issues (Firdaus and Rahman, 2012). However, the chattering 
phenomenon following the use of SMC is still an issue to be resolved. Some methods have been proposed to eliminate this phenomenon. These include quasi sliding mode control (QuasiSMC) (Shtessel et al., 2010); interval type-2 fuzzy sliding mode control (IT2FSMC) (Firdaus and Tokhi, 2016); high order sliding mode control (HOSMC) (Ghabi, 2018); super-twisting algorithm of sliding mode control (STASMC) (Ibarra and Castillo, 2017); and dynamic sliding mode control (DSMC) (Liu and Wang, 2011). Although these methods can eliminate the problem of chattering, they have their associated shortcomings. The QuasiSMC method will not be effective to reduce chattering when systems require high accuracy control signals. IT2FSMC as model-free approach maybe a better method to deal with non-linear systems, multivariable, and uncertainty models caused by imprecision or unknown physical parameters. However, this method still has an issue of computational time in type reduction process which may not be realistic in real-time applications. HOSMC can reduce oscillation phenomenon in SMC adequately, but this method still cannot address the problem in $n$-th derivative states. Although, STASMC is more effective than HOSMC in eliminating chattering, the method still cannot compensate for uncertainty and disturbance on state variables. Among the methods, DSMC has a better way to deal with measurement noise as well as chattering reduction.

In addition to selecting a proper controller to deal with the complexity of quadcopter, the availability of information on required states is a matter for SMC to be implemented experimentally. Consequently, the use of an observer can be a solution to address such issue. Previous works have included the use of different types of observer methods in quadcopter UAVs. These include nonlinear observer (NO) (Bouadi and Tadjine, 2007, Elamine et al., 2013); sliding mode observer(SMO) (Firdaus and Tokhi, 2015, Gonzalez-Hernandez et al., 2017, Boiko and Chehadeh, 2018); Thau observer Cen et al. (2014) and Kalman filter(KF) Liu et al. (2013), Sebesta and Boizot (2014). Although results show that the methods can improve the performance of the controller, some shortcomings still accompany such methods. The fundamental disadvantage of $\mathrm{NO}$ is connected to the expanding power of the gain parameter which led to hard task numerical execution when the gain and the system order are very large (Astolfi and Marconi, 2015). Furthermore, a comparative study between SMO and KF was conducted by Chen and Dunnigan (2002). They pointed out that SMO is more robust than $\mathrm{KF}$ in terms of parameter uncertainty and noise cancellation. Meanwhile, Thau observer may not deal with error model of the system (Schröder et al., 2000). However, although SMO has shown better performance, the observer still suffers from chattering, and this affects its overall performance with measurement noise. The way to address the chattering problem in SMO is much similar with SMC.
This paper proposes performance enhancement of DSMC by employing integral term and set-point weighting function to the method to reduce steady-state error and overshoot. In addition, this paper presents investigation and analyses of several performance assessment of SMO-based observers, including quasi sliding mode observer (QuasiSMO), interval type2 fuzzy sliding mode observer (IT2FSMO), supertwisting algorithm of sliding mode observer (STASMO), and higher order sliding mode observer (HOSMO). Comparative performance assessment of the observers is carried out through numerical simulations within the framework of quadcopter UAV control. Moreover, the performance comparison between SMO-based observer and EKF is presented to highlight the robustness of each method.

The rest of the paper is structured as follows: Section 2 describes dynamic model of the quadcopter, Section 3 presents the general design of quadcopter control and observer, Integral DSMC and SMOs are presented in section 4 and 5 respectively. Numerical simulation results demonstrating performance of the approaches are presented and discussed in Section 6 and the paper is concluded in Section 7.

\section{Quadcopter system model}

A quadcopter UAVs as illustrated in Figure 1, is outlined with two clockwise pivoting rotors $(2,4)$, and two counter-clockwise turning rotors $(1,3)$, so that by accelerating the rotor at the same rate will build the height of rotorcraft, and the other way around. Forward movement can be accomplished by lessening the rotor speed (1) and increasing the speed of rotor (3) at the same time. The similar way should be possible for reverse movement, rightward, and leftward development. Moreover, the yaw motion can occur by increasing or reducing the clockwise rotors speed of the quadcopter. Nevertheless, the total thrust force of the rotors must have the same value to keep up the height level of the vehicle.

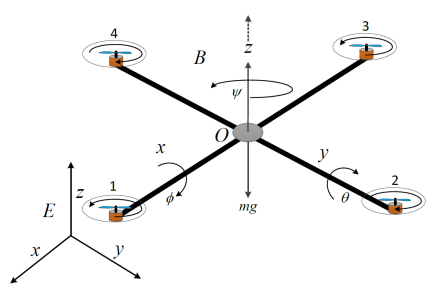

Figure 1. Quadcopter UAVs orientation

The framework of the vehicle can be isolated into two sections, to be specific the "body frame" B-(Oxyz), and the "earth frame" E-(Oxyz). Meanwhile $m, g$, and $l$ designate the the rotorcraft mass, gravity acceleration, and the distance of every rotor to centre of gravity of rotorcraft respectively.

The orientation of the rotocraft from body frame to earth frame is represented by rotation matrices of 
$R: E \longrightarrow B$, where these matrices depend on the Euler angle $(\phi, \theta, \psi)$. Final rotation matrix from the earth frame to the body frame should be transposed to get transformation from body frame to earth frame as (Elkholy, 2014),

$$
R=\left[\begin{array}{ccc}
c \theta c \psi & s \phi s \theta c \psi-c \phi s \psi & s \phi s \psi+c \phi s \theta c \psi \\
c \theta s \psi & c \phi c \psi+s \phi s \psi s \theta & c \phi s \psi s \theta-s \phi c \psi \\
-s \theta & s \phi c \theta & c \phi c \theta
\end{array}\right]
$$

The angles $\phi, \theta$, and $\psi$ denote roll, pitch, and yaw respectively, while $s, c$, and $t$ represent sine, cos, and tangent respectively. The movement of roll $(\phi)$, pitch $(\theta)$ and yaw $(\psi)$ are bounded as follows: $(-\pi / 2<\phi<\pi / 2)$, $(-\pi / 2<\theta<\pi / 2)$ and $(-\pi<\psi<\pi)$ respectively.

The equations of rotational movement in the body frame are obtained by utilizing the general equation of Newton - Euler method as presented,

$$
J \dot{\omega}+\omega \times J \omega+M_{G}=M_{B}
$$

where $J, \omega, M_{G}$, and $M_{B}$ denote the inertia matrix of quadcopter, body angular velocity, gyroscopic moments because of inertia produced by rotors motion, and moments working on the quadcopter in the body frame respectively. Furthermore, the gyroscopic moments are defined as $\omega \times\left[\begin{array}{lll}0 & 0 & J_{r} \Omega_{r}\end{array}\right]^{T}$, where $J_{r}$ and $\Omega_{r}=$ $-\Omega_{1}+\Omega_{2}-\Omega_{3}+\Omega_{4}$ denote rotors' inertia and residual of angular rotors velocity respectively. Quadcopter is designed to be symmetric so that its inertial matrix for each component $\mathrm{x}, \mathrm{y}$ and $\mathrm{z}$ is diagonal as $J=\operatorname{diag}\left[I_{x x} I_{y y}\right.$ $\left.I_{z z}\right]$ where $I_{x x}, I_{y y}$ and $I_{z z}$ are the moments of inertia for each axis in the body frame reference.

There are two physical effects which produced by rotors forces, namely aerodynamic force $F_{i}$ and aerodynamic moments $M_{i}$. The aerodynamic force is the force applied on a body of rotorcraft by the air in which the body is submerged, and is because of the relative motion between the body and the air generated by rotors. Meanwhile, aerodynamic moment is the reactive torque caused by the rotor motion on the air. Those aerodynamic effects are defined as,

$$
\begin{aligned}
& F_{i}=K_{f} \Omega_{i}^{2} \\
& M_{i}=K_{M} \Omega_{i}^{2}
\end{aligned}
$$

where $K_{f}$ and $K_{M}$ are the aerodynamic force and moment constants respectively and $\Omega_{i}$ is the angular velocity of rotor $i$.

In the body frame $x$-axis, the rolling torque is derived by multiplying $F_{2}$ with the arm length $l$ of rotorcraft. This produces a negative moment in the $x$-axis, while in the same way, $F_{4}$ produces a positive moment. Hence, the rolling torque is defined as,

$$
\begin{aligned}
M_{\phi} & =-F_{2} l+F_{4} l \\
& =l K_{f}\left(-\Omega_{2}^{2}+\Omega_{4}^{2}\right)
\end{aligned}
$$

Furthermore, in the body frame $y$-axis, the thrust of rotor 1 produces a positive moment, while rotor 3 delivers a negative moment in the $y$-axis. Therefore, the pitching torque is defined as,

$$
\begin{aligned}
M_{\theta} & =F_{1} l-F_{3} l \\
& =l K_{f}\left(\Omega_{1}^{2}-\Omega_{3}^{2}\right)
\end{aligned}
$$

For the motion in the body frame $z$-axis, The moment caused by the rotors' rotation is presented in equation (4). Thus, the yawing torque in the body frame $z$-axis is defined as,

$$
\begin{aligned}
M_{\psi} & =M_{1}-M_{2}+M_{3}-M_{4} \\
& =K_{M}\left(\Omega_{1}^{2}-\Omega_{2}^{2}+\Omega_{3}^{2}-\Omega_{4}^{2}\right)
\end{aligned}
$$

In vector form, the moments expressed in equations (5), (6) and (7) can be presented as,

$$
M_{B}=\left[\begin{array}{c}
l K_{f}\left(-\Omega_{2}^{2}+\Omega_{4}^{2}\right) \\
l K_{f}\left(\Omega_{1}^{2}-\Omega_{3}^{2}\right) \\
K_{M}\left(\Omega_{1}^{2}-\Omega_{2}^{2}+\Omega_{3}^{2}-\Omega_{4}^{2}\right)
\end{array}\right]
$$

where $l$ is the distance between rotor and centre of gravity of quadcopter.

The translation equations of quadcopter motion in the earth frame are derived by utilizing Newton's second law as expressed as,

$$
m \ddot{r}=\left[\begin{array}{c}
0 \\
0 \\
-m g
\end{array}\right]+R F_{B}
$$

where $r=\left[\begin{array}{lll}x & y & z\end{array}\right]^{T}, m$, and $g$ represent quadrotor's distance from the Earth frame, quadrotor's mass, and gravitational acceleration respectively, while $R$ and $F_{B}$ denote rotation matrix and non-gravitational forces working on the quadrotor in the body frame.

When quadcopter is the steady-state condition, the only non-gravitational forces working on it is the thrust generated by propellers motions as defined in (3). Thus, the non-gravitational forces working on the quadcopter, $F_{B}$, is presented as,

$$
F_{B}=\left[\begin{array}{c}
0 \\
0 \\
K_{f}\left(\Omega_{1}^{2}+\Omega_{2}^{2}+\Omega_{3}^{2}+\Omega_{4}^{2}\right)
\end{array}\right]
$$

A control input vector of quadcopter is defined as,

$$
u=\left[\begin{array}{llll}
u_{1} & u_{2} & u_{3} & u_{4}
\end{array}\right]^{T}
$$

where

$$
\begin{aligned}
& u_{1}=K_{f}\left(\Omega_{1}^{2}+\Omega_{2}^{2}+\Omega_{3}^{2}+\Omega_{4}^{2}\right) \\
& u_{2}=l K_{f}\left(-\Omega_{2}^{2}+\Omega_{4}^{2}\right) \\
& u_{3}=l K_{f}\left(\Omega_{1}^{2}-\Omega_{3}^{2}\right) \\
& u_{4}=K_{M}\left(\Omega_{1}^{2}-\Omega_{2}^{2}+\Omega_{3}^{2}-\Omega_{4}^{2}\right)
\end{aligned}
$$

Furthermore, by using equations (2), (8), and (12) the angular accelerations of the rotorcraft can be presented as,

$$
\begin{aligned}
& \ddot{\phi}=\dot{\psi} \dot{\theta}\left(\frac{I_{y y}-I_{z z}}{I_{x x}}\right)-\frac{J_{r}}{I_{x x}} \dot{\theta} \Omega_{r}+\frac{l}{I_{x x}} u_{2} \\
& \ddot{\theta}=\dot{\phi} \dot{\psi}\left(\frac{I_{z z-I_{x x}}}{I_{y y}}\right)+\frac{J_{r}}{I_{y y}} \dot{\phi} \Omega_{r}+\frac{l}{I_{y y}} u_{3} \\
& \ddot{\psi}=\dot{\phi} \dot{\theta}\left(\frac{I_{x x}-I_{y y}}{I_{z z}}\right)+\frac{1}{I_{z z}} u_{4}
\end{aligned}
$$

The translational equation can be obtained by using equations (9), (10), and (12),

$$
\begin{aligned}
\ddot{x} & =\frac{u_{1}}{m}(\sin \phi \sin \psi+\cos \phi \sin \theta \cos \psi) \\
\ddot{y} & =\frac{u_{1}}{m}(\cos \phi \sin \psi \sin \theta-\sin \phi \cos \psi) \\
\ddot{z} & =-g+\frac{u_{1}}{m}(\cos \phi \cos \theta)
\end{aligned}
$$




\section{Quadcopter control and observer design}

Refers to the system dynamics defined in equations (13) and (14), it is noted that the system is nonlinear and comprises two subsystems, namely fully-actuated and underactuated subsystem. Therefore, the architecture of the controller can be designed as Figure 2, where $x_{d}, y_{d}$ and $z_{d}$ represent the desired positions of the quadcopter in the $\mathrm{x}, \mathrm{y}$ and $\mathrm{z}$ axes respectively and $\phi_{d}, \theta_{d}$ and $\psi_{d}$ denote the reference roll, pitch and yaw angles respectively.

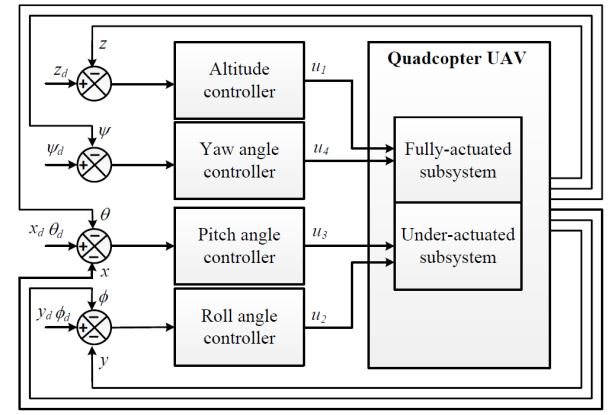

Figure 2. Quadcopter controller architecture

In this work, set-point weighting-based dynamic integral sliding mode control (SDISMC) is employed to control the altitude and attitude of the quadcopter system. Meanwhile for performance comparison purposes, sliding mode-based observers including QuasiSMO, IT2FLSMO, STASMO, and HOSMO are used to estimate unmeasured states of the vehicle which is required for control process. In addition, the performance comparison between sliding mode-based observers and EKF is undertaken also to highlight the robustness of each method. Thus, the overall control system is shown as in Figure 3.

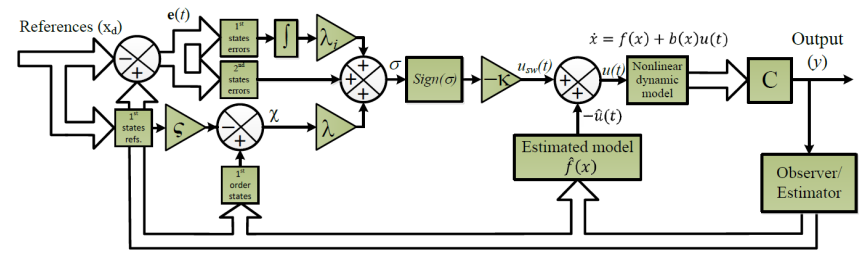

Figure 3. Block diagram of overall control system

\section{Set-point weighting-based integral sliding mode control}

dynamic

Sliding mode control is a prominent robust control approach which gives an efficient way to deal with the problem of keeping up stability and consistent performance in the face of modelling imperfection. In order to account for the presence of disturbance and imperfection model, the control law should be discontinuous across $\sigma(t)$. However, this will result in chattering as seen in Figure 4 due to the imperfection implementation of such control law. This phenomenon is not expected in practice because it involves high control activities.

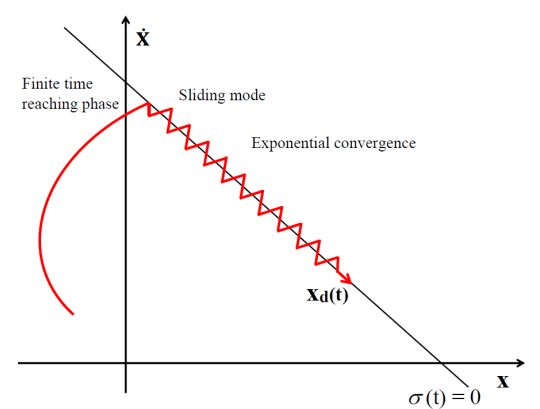

Figure 4. Block diagram of overall control system

One of the ways to address chattering issue is by developing dynamic sliding surface. The method construct a new sliding function from a conventional switching surface of SMC. The dynamic sliding surface is with respect to the first or high-order derivative in the control input. Additionally, it can move the discontinuous terms into the first-order or high-order derivative in the control law. Therefore, a continuous DSMC rule is obtained and the chattering issue can be lessened adequately (Liu and Wang, 2011).

\subsection{Integral sliding surface design}

General form of nonlinear system can be defined as

$$
\begin{aligned}
& \dot{x}_{1}=x_{2} \\
& \dot{x}_{2}=f(\mathbf{x}, t)+b(\mathbf{x}, t) u(t)+d(t) \\
& y=x_{1}
\end{aligned}
$$

where $\mathbf{x}=\left[\begin{array}{ll}x_{1} & x_{2}\end{array}\right]$ is the state vector, $x_{1}$ and $x_{2}$ are system states, $y$ is output, $u(t)$ is control input, $f(\mathbf{x}, t)$ and $b(\mathbf{x}, t)$ represent general nonlinear functions. These functions are not accurately known, but the degree of inaccuracy on those function are upper bounded by a known continuous function of $\mathbf{x} . d(t)$ is uncertain, and $|d(t)| \leqslant D_{0},|\dot{d}(t)| \leqslant D$. The control input role is to convey the state $\mathbf{x}$ to track a desired state $\mathbf{x}_{d}$ despite of model imperfection on $f(\mathbf{x}, t)$ and $b(\mathbf{x}, t)$.

A time varying integral switching surface $s(t)$ is defined in the state space $\mathbf{R}^{n}$ by equating the variable $s(\mathbf{x} ; t)$ to zero as expressed,

$$
s(\mathbf{x} ; t)=\left(\frac{d}{d t}+c\right)^{n-1} \mathbf{e}(t)+\lambda_{i} \int_{0}^{\infty} \mathbf{e}(t) d t
$$

where, $c, \lambda_{i}>0$ must be Hurwitz, taken to be the bandwidth of the system, and $\mathbf{e}(t)=\mathbf{y}(t)-\mathbf{x}_{d}(t)$ is the error in the output state, while $\mathbf{x}_{d}(t)$ is the expected state. An integral term of tracking error is introduced into equation to reduce steady-state errors.

\subsection{Set-point weighting}

Employing integral term in SMC is believed to improve the controller performance in reducing steady-state error. However, any of the following issues can generate an overshoot in output variable:

- $|\mathbf{e}(t)|>\left|\mathbf{e}_{s s}\right|$, where $\mathbf{e}_{s s}$ is steady-state error of $\mathbf{e}(t)$ 
- $\frac{\mathbf{e}(t)}{|\mathbf{e}(t)|} \neq \frac{\mathbf{e}_{s s}}{\left|\mathbf{e}_{s s}\right|}$

Additionally, considering typical feedback controller design, such as PID, the issue will be how to achieve fast reference tracking and reject disturbance at the same time. Generally, high-gain control parameters are required to deal with load disturbance. However at the same time, it will lead to oscillation or high overshoot of step response. This problem can be solved by introducing set-point weighting to increase the frequency of Zero, and subsequently diminish the overshoot in the output (Visioli, 2006).

This approach is adopted in this work to handle the problem with integral SMC to reduce overshoot in output states while maintaining robustness of the controller. Therefore, the new sliding mode approach by applying set-point weighting can be designed as shown in Figure 3. Accordingly, a new error $\chi(t)$ for proportional action is presented as

$$
\chi=e(t)+r(1-\varsigma)
$$

where $r$ is reference and $\varsigma$ is constant between 0 and 1. Reducing $\varsigma$ will lead to decreasing overshoot and rise time, and vice versa. Consequently, the new sliding surface is defined as,

$$
s(\mathbf{x} ; t)=\left(\frac{d}{d t}+c\right)^{n-1} \chi(t)+\lambda_{i} \int_{0}^{\infty} \mathbf{e}(t) d t
$$

\subsection{Control law}

Consider the general form on nonlinear system (15), and integral sliding surface (18). Hence,

$$
\begin{aligned}
& \dot{s}=\ddot{e}+c \dot{e}+\lambda_{i} e \\
& \dot{s}=f(x)+b(x) u(t)+d(t)-\ddot{x}_{d}+c \dot{e}+\lambda_{i} e
\end{aligned}
$$

Furthermore, a new dynamic sliding manifold is constructed as(Liu and Wang, 2011)

$$
\sigma=\dot{s}+\lambda s
$$

where $\lambda>0$ must be Hurwitz. When $\sigma=0, \dot{\sigma}=0$ is a asymptotically stable, therefore, $e \longrightarrow 0$ and $\dot{e} \longrightarrow 0$. From equation (20), stability analysis is expressed as,

$$
\begin{aligned}
\sigma & =\dot{s}+\lambda s \\
& =f(x)+b(x) u(t)+d(t)-\ddot{x}_{d}+c \dot{e}+\lambda_{i} e+\lambda s
\end{aligned}
$$

Thus,

$$
\begin{aligned}
\dot{\sigma}= & \dot{f}(x)+\dot{b}(x) u(t)+b(x) \dot{u}(t)+\dot{d}(t)-\dddot{x}_{d} \\
& +c \ddot{e}+\lambda_{i} \dot{e}+\lambda \dot{s} \\
= & \dot{f}(x)-(c+\lambda) \ddot{y}_{d}-\dddot{y}_{d}+\dot{d}(t)+(c+\lambda) d(t) \\
& +(\dot{b}(x)+c b(x)+\lambda b(x)) u(t)+(c+\lambda) f(x) \\
& +b(x) \dot{u}(t)+\lambda \lambda_{i} e+\lambda c \dot{e}+\lambda_{i} \dot{e}
\end{aligned}
$$

Select the dynamic control law as,

$$
\begin{aligned}
\dot{u}(t)= & \frac{1}{b(x)}\left(-\dot{f}(x)+(c+\lambda) \ddot{y}_{d}+\dddot{y}_{d}-(\dot{b}(x)\right. \\
& +c b(x)+\lambda b(x)) u(t)-(c+\lambda) f(x) \\
& \left.-\lambda c \dot{e}-\lambda \lambda_{i} e-k \frac{\sigma}{|\sigma|}-\mu \sigma\right)
\end{aligned}
$$

where $k, \mu>0$. Stability analysis can be expressed as

$$
\dot{\sigma}=\dot{d}(t)+(c+\lambda) d(t)-k \frac{\sigma}{|\sigma|}
$$

Let $k>D+(c+\lambda) D_{0}$, therefore,

$$
\begin{aligned}
\sigma \dot{\sigma}= & \sigma\left(\dot{d}(t)+(c+\lambda) d(t)-k \frac{\sigma}{|\sigma|}\right) \\
= & \sigma(\dot{d}(t)+(c+\lambda) d(t))-k|\sigma| \leqslant(D \\
& \left.+(c+\lambda) D_{0}\right) \sigma-k|\sigma|<0
\end{aligned}
$$

From (18), sliding surface for the quadcopter UAVs are defined as

$$
\begin{aligned}
& s_{\phi}=c_{\phi} \chi_{\phi}+\dot{e}_{\phi}+\lambda_{i \phi} \int_{0}^{\infty} e_{\phi} d t \\
& s_{\theta}=c_{\theta} \chi_{\theta}+\dot{e}_{\theta}+\lambda_{i \theta} \int_{0}^{\infty} e_{\theta} d t \\
& s_{\psi}=c_{\psi} \chi_{\psi}+\dot{e}_{\psi}+\lambda_{i \psi} \int_{0}^{\infty} e_{\psi} d t \\
& s_{z}=c_{z} \chi_{z}+\dot{e}_{z}+\lambda_{i z} \int_{0}^{\infty} e_{z} d t \\
& s_{x}=c_{x} \chi_{x}+\dot{e}_{x}+\lambda_{i x} \int_{0}^{\infty} e_{x} d t \\
& s_{y}=c_{y} \chi_{y}+\dot{e}_{y}+\lambda_{i y} \int_{0}^{\infty} e_{y} d t
\end{aligned}
$$

Referring to equations (19) and (26), the derivative of sliding surface is expressed as

$$
\begin{aligned}
& \dot{s}_{\phi}=c_{\phi} \dot{e}_{\phi}+f_{\phi}+b_{\phi} u_{2}-\ddot{\phi}_{d}+\lambda_{i \phi} e_{\phi} \\
& \dot{s}_{\theta}=c_{\theta} \dot{e}_{\theta}+f_{\theta}+b_{\theta} u_{3}-\ddot{\theta}_{d}+\lambda_{i \theta} e_{\theta} \\
& \dot{s}_{\psi}=c_{\psi} \dot{e}_{\psi}+f_{\psi}+b_{\psi} u_{4}-\ddot{\psi}_{d}+\lambda_{i \psi} e_{\psi} \\
& \dot{s}_{z}=c_{z} \dot{e}_{z}+f_{z}+b_{z} u_{1}-\ddot{z}_{d}+\lambda_{i z} e_{z} \\
& \dot{s}_{x}=c_{x} \dot{e}_{x}+f_{x}+b_{x} u_{1}-\ddot{x}_{d}+\lambda_{i x} e_{x} \\
& \dot{s}_{y}=c_{y} \dot{e}_{y}+f_{y}+b_{y} u_{1}-\ddot{y}_{d}+\lambda_{i y} e_{y}
\end{aligned}
$$

Furthermore, the new sliding manifold is constructed as

$$
\begin{aligned}
\sigma_{\phi} & =\dot{s}_{\phi}+\lambda_{\phi} s_{\phi} \\
\sigma_{\theta} & =\dot{s}_{\theta}+\lambda_{\theta} s_{\theta} \\
\sigma_{\psi} & =\dot{s}_{\psi}+\lambda_{\psi} s_{\psi} \\
\sigma_{z} & =\dot{s}_{z}+\lambda_{z} s_{z} \\
\sigma_{x} & =\dot{s}_{x}+\lambda_{x} s_{x} \\
\sigma_{y} & =\dot{s}_{y}+\lambda_{y} s_{y}
\end{aligned}
$$


When $\sigma=0, \quad \dot{\sigma}=0$ is a asymptotically stable and referring to (23), the control input of quadcopter UAVs can be defined as

$$
\begin{aligned}
u_{1}= & \int_{0}^{\infty} \frac{1}{b_{z}}\left(-\dot{f}_{z}+\left(c_{z}+\lambda_{z}\right) \ddot{z}_{d}+\dddot{z}_{d}-\left(\dot{b}_{z}+c_{z} b_{z}\right.\right. \\
& \left.+\lambda_{z} b_{z}\right) u_{1}-\left(c_{z}+\lambda_{z}\right) f_{z}-\lambda_{z} c_{z} \dot{e}_{z}-\lambda_{i z} \dot{e}_{z} \\
& \left.-\lambda_{z} \lambda_{i z} e_{z}-k_{z} \frac{\sigma_{z}}{\left|\sigma_{z}\right|}-\mu_{z} \sigma_{z}\right) d t \\
u_{2}= & \int_{0}^{\infty} \frac{1}{b_{\phi}}\left(-\dot{f}_{\phi}+\left(c_{\phi}+\lambda_{\phi}\right) \ddot{\phi}_{d}+\dddot{\phi}_{d}-\left(\dot{b}_{\phi}\right.\right. \\
& \left.+c_{\phi} b_{\phi}+\lambda_{\phi} b_{\phi}\right) u_{2}-\left(c_{\phi}+\lambda_{\phi}\right) f_{\phi}-\lambda_{\phi} c_{\phi} \dot{e}_{\phi} \\
& \left.-\lambda_{i \phi} \dot{e}_{\phi}-\lambda_{\phi} \lambda_{i \phi} e_{\phi}-k_{\phi} \frac{\sigma_{\phi}}{\left|\sigma_{\phi}\right|}-\mu_{\phi} \sigma_{\phi}\right) d t \\
u_{3}= & \int_{0}^{\infty} \frac{1}{b_{\theta}}\left(-\dot{f}_{\theta}+\left(c_{\theta}+\lambda_{\theta}\right) \ddot{\theta}_{d}+\dddot{\theta}_{d}-\left(\dot{b}_{\theta}\right.\right. \\
& \left.+c_{\theta} b_{\theta}+\lambda_{\theta} b_{\theta}\right) u_{3}-\left(c_{\theta}+\lambda_{\theta}\right) f_{\theta}-\lambda_{\theta} c_{\theta} \dot{e}_{\theta} \\
& \left.-\lambda_{i \theta} \dot{e}_{\theta}-\lambda_{\theta} \lambda_{i \theta} e_{\theta}-k_{\theta} \frac{\sigma_{\theta}}{\left|\sigma_{\theta}\right|}-\mu_{\theta} \sigma_{\theta}\right) d t \\
u_{4}= & \int_{0}^{\infty} \frac{1}{b_{\psi}}\left(-\dot{f}_{\psi}+\left(c_{\psi}+\lambda_{\psi}\right) \ddot{\psi}_{d}+\dddot{\psi}_{d}-\left(\dot{b}_{\psi}\right.\right. \\
& \left.+c_{\psi} b_{\psi}+\lambda_{\psi} b_{\psi}\right) u_{4}-\left(c_{\psi}+\lambda_{\psi}\right) f_{\psi}-\lambda_{\psi} c_{\psi} \dot{e}_{\psi} \\
& \left.-\lambda_{i \psi} \dot{e}_{\psi}-\lambda_{\psi} \lambda_{i \psi} e_{\psi}-k_{\psi} \frac{\sigma_{\psi}}{\left|\sigma_{\psi}\right|}-\mu_{\psi} \sigma_{\psi}\right) d t
\end{aligned}
$$

Furthermore, the calculation of the desired pitch $\left(\theta_{d}\right)$ and roll $\left(\phi_{d}\right)$ angles with $x$ and $y$ axes errors are presented as:

$$
\begin{aligned}
\theta_{d}= & \int_{0}^{\infty} \frac{1}{u_{1}}\left(-\dot{f}_{x}+\left(c_{x}+\lambda_{x}\right) \ddot{x}_{d}+\dddot{x}_{d}-\left(\dot{u}_{1}\right.\right. \\
& \left.+c_{x} u_{1}+\lambda_{x} u_{1}\right) \theta_{d}-\left(c_{x}+\lambda_{x}\right) f_{x}-\lambda_{x} c_{x} \dot{e}_{x} \\
& \left.-\lambda_{i x} \dot{e}_{x}-\lambda_{x} \lambda_{i x} e_{x}-k_{x} \frac{\sigma_{x}}{\left|\sigma_{x}\right|}-\mu_{x} \sigma_{x}\right) d t \\
\phi_{d}= & -\int_{0}^{\infty} \frac{1}{u_{1}}\left(-\dot{f}_{y}+\left(c_{y}+\lambda_{y}\right) \ddot{y}_{d}+\dddot{y}_{d}-\left(\dot{u}_{1}\right.\right. \\
& \left.+c_{y} u_{1}+\lambda_{y} u_{1}\right) \phi_{d}-\left(c_{y}+\lambda_{y}\right) f_{y}-\lambda_{y} c_{y} \dot{e}_{y} \\
& \left.-\lambda_{i y} \dot{e}_{y}-\lambda_{y} \lambda_{i y} e_{y}-k_{y} \frac{\sigma_{y}}{\left|\sigma_{y}\right|}-\mu_{y} \sigma_{y}\right) d t
\end{aligned}
$$

\section{Sliding mode observer}

$\mathrm{SMO}$, as a nonlinear estimator, has interesting properties in that the capacity to create a sliding motion on the estimation error, and to generates estimated states precisely equivalent with actual output of the plant. Moreover, the estimator technique is considered as the forefront of robust approach for state and parameter estimation by demonstrating the ability to handle model errors and nonlinear systems.

\subsection{Basic observer design}

Consider the general nonlinear system as expressed in (15). If the measured state is $x_{1}$, an observer structure is expressed as(Slotine et al., 1986),

$$
\begin{aligned}
& \dot{\hat{x}}_{1}=-\alpha_{1} e_{1}+\hat{x}_{2}-k_{1} \frac{e_{1}}{\left|e_{1}\right|} \\
& \dot{\hat{x}}_{2}=-\alpha_{2} e_{1}+\hat{f}+\hat{b} u-k_{2} \frac{e_{1}}{\left|e_{1}\right|}
\end{aligned}
$$

where $e_{1}=\hat{x}_{1}-x_{1}, \hat{x}_{i}$, and $u$ represent estimation error, estimated state of $x_{i}$, and control law respectively, while $\hat{f}$ and $\hat{b}$ denote the best estimated models of $f$ and $b$ respectively, and the constants $\alpha_{i}$ are selected as in a Luenberger estimator (which relate to $k_{1}=0, k_{2}=0$ ) so as to locate the poles of the linearised system at expected places $-\zeta_{i}$. The sliding gain $k_{1}$ is selected as a bound on estimated state error of $x_{2}$ in the steady state condition, and $k_{2}$ is selected to be bigger than modelling errors. The resulting error dynamics of the approach can be composed as (Slotine et al., 1986),

$$
\begin{aligned}
& \dot{e_{1}}=-\alpha_{1} e_{1}+e_{2}-k_{1} \frac{e_{1}}{\left|e_{1}\right|} \\
& \dot{e_{2}}=-\alpha_{2} e_{1}+\Delta f-k_{2} \frac{e_{1}}{\left|e_{1}\right|}
\end{aligned}
$$

The value of $\Delta f=\hat{f}-f$ relies on the computational intricacy and the modelling effort allowed in the estimator. In this research, dynamic uncertainty $\Delta f$ is assumed to be bounded explicitly. For simplicity, the known nonlinear terms may likewise be treated as bounded error (using known bounds on the actual system state) and incorporated in $\Delta f$. The effect of $\Delta f$ is compensated by exploiting this knowledge of its (generally time-varying) bound.

\subsection{Observer design for quadcopter UAVs}

It is important to determine states vector of quadcopter before designing states observer for the system. Hence, the state vector of the system is defined as,

$$
X=\left[\begin{array}{llllllllllll}
x_{1} & x_{2} & x_{3} & x_{4} & x_{5} & x_{6} & x_{7} & x_{8} & x_{9} & x_{10} & x_{11} & x_{12}
\end{array}\right]^{T}
$$

The states vector is mapped to the degrees of freedom of the quadcopter, as expressed,

$$
X=\left[\begin{array}{llllllllllll}
\phi & \dot{\phi} & \theta & \dot{\theta} & \psi & \dot{\psi} & z & \dot{z} & x & \dot{x} & y & \dot{y}
\end{array}\right]^{T}
$$

The measured states of the system are roll $\left(x_{1}\right)$, pitch $\left(x_{3}\right)$ and yaw $\left(x_{5}\right)$ angles, and linear movements in $z\left(x_{7}\right)$, $x\left(x_{9}\right)$, and $y\left(x_{11}\right)$ axes. Basically, SMC as state feedback control system needs the availability of full state to control the system. Thefore, the observer plays a critical role in estimating the velocity of the rotorcraft, such as $x_{2}, x_{4}, x_{6}, x_{8}, x_{10}$, and $x_{12}$.

Considering the dynamic system of quadcopter (13) and (14), state vectors and second order observer structure (31), the states estimator for quadcopter UAVs systems is presented as, 
Firdaus, A. R. and Tokhi, M. O.

$$
\begin{aligned}
& \dot{\hat{x}}_{1}=-\alpha_{1} e_{1}+\hat{x}_{2}-k_{1} \frac{e_{1}}{\left|e_{1}\right|} \\
& \dot{\hat{x}}_{2}=-\alpha_{2} e_{1}+\hat{x}_{4} \hat{x}_{6}\left(\frac{I_{y}-I_{z}}{I_{x}}\right)+\frac{l}{I_{x}} u_{2}-k_{2} \frac{e_{1}}{\left|e_{1}\right|} \\
& \dot{\hat{x}}_{3}=-\alpha_{1} e_{3}+\hat{x}_{4}-k_{1} \frac{e_{3}}{\left|e_{3}\right|} \\
& \dot{\hat{x}}_{4}=-\alpha_{2} e_{3}+\hat{x}_{2} \hat{x}_{6}\left(\frac{I_{z}-I_{x}}{I_{y}}\right)+\frac{l}{I_{y}} u_{3}-k_{2} \frac{e_{3}}{\left|e_{3}\right|} \\
& \dot{\hat{x}}_{5}=-\alpha_{1} e_{5}+\hat{x}_{6}-k_{1} \frac{e_{5}}{\left|e_{5}\right|} \\
& \dot{\hat{x}}_{6}=-\alpha_{2} e_{5}+\hat{x}_{2} \hat{x}_{4}\left(\frac{I_{x}-I_{y}}{I_{z}}\right)+\frac{1}{I_{z}} u_{4}-k_{2} \frac{e_{5}}{\left|e_{5}\right|} \\
& \dot{\hat{x}}_{7}=-\alpha_{1} e_{7}+\hat{x}_{8}-k_{1} \frac{e_{7}}{\left|e_{7}\right|} \\
& \dot{\hat{x}}_{8}=-\alpha_{2} e_{7}-g+\frac{c x_{1} c x_{3}}{m} u_{1}-k_{2} \frac{e_{7}}{\left|e_{7}\right|} \\
& \dot{\hat{x}}_{9}=-\alpha_{1} e_{9}+\hat{x}_{10}-k_{1} \frac{e_{9}}{\left|e_{9}\right|} \\
& \dot{\hat{x}}_{10}=-\alpha_{2} e_{9}+\frac{c x_{1} s x_{3} c x_{5}+s x_{1} s x_{5}}{m} u_{1}-k_{2} \frac{e_{9}}{\left|e_{9}\right|} \\
& \dot{\hat{x}}_{11}=-\alpha_{1} e_{11}+\hat{x}_{12}-k_{1} \frac{e_{11}}{\left|e_{11}\right|} \\
& \dot{\hat{x}}_{12}=-\alpha_{2} e_{11}+\frac{c x_{1} s x_{3} s x_{5}-s x_{1} c x_{5}}{m} u_{1}-k_{2} \frac{e_{11}}{\left|e_{11}\right|}
\end{aligned}
$$

where $e=\hat{x}-x$ is the estimated states error, $\hat{x}$ represent estimated states, and $\alpha_{i}, k_{i}>0$.

\subsection{Chattering avoidance: elimination and attenuation}

The discontinuous term in SMO may lead to chattering phenomenon. Despite the fact that this issue is just connected to numerical execution instead of "hard" mechanical restrictions, the chattering is still harmful for obtaining high accuracy estimated states. Therefore, some studies have been conducted to reduce or eliminate such phenomenon, including QuasiSMO (Shtessel et al., 2010), IT2FSMO (Firdaus and Tokhi, 2015), HOSMO (Gonzalez-Hernandez et al., 2017, Skender et al., 2017), and STASMO (Salgado et al., 2011).

\subsubsection{Chattering elimination: quasi-sliding mode observer}

The approach to eliminate the chattering using QuasiSMO is to replace the discontinuous term $k \frac{e}{|e|}$ with continuous term, expressed as (Shtessel et al., 2010),

$$
\frac{e}{\mid e} \rightarrow \frac{e}{|e|+\varepsilon}
$$

where $\varepsilon$ is a small positive constant. It can be seen that,

$$
\lim _{\varepsilon \longrightarrow 0} \frac{e}{|e|+\varepsilon}=\frac{e}{|e|}
$$

for $e \neq 0$. The value of $\varepsilon$ should be selected to meet a smooth control signals while maintaining an ideal performance of control method.

Hence, the observer can be presented as

$$
\begin{aligned}
& \dot{\hat{x}}_{1}=-\alpha_{1} e_{1}+\hat{x}_{2}-k_{1} \frac{e_{1}}{\left|e_{1}\right|+\varepsilon} \\
& \dot{\hat{x}}_{2}=-\alpha_{2} e_{1}+\hat{x}_{4} \hat{x}_{6}\left(\frac{I_{y}-I_{z}}{I_{x}}\right)+\frac{l}{I_{x}} u_{2}-k_{2} \frac{e_{1}}{\left|e_{1}\right|+\varepsilon} \\
& \dot{\hat{x}}_{3}=-\alpha_{1} e_{3}+\hat{x}_{4}-k_{1} \frac{e_{3}}{\left|e_{3}\right|+\varepsilon} \\
& \dot{\hat{x}}_{4}=-\alpha_{2} e_{3}+\hat{x}_{2} \hat{x}_{6}\left(\frac{I_{z}-I_{x}}{I_{y}}\right)+\frac{l}{I_{y}} u_{3}-k_{2} \frac{e_{3}}{\left|e_{3}\right|+\varepsilon} \\
& \dot{\hat{x}}_{5}=-\alpha_{1} e_{5}+\hat{x}_{6}-k_{1} \frac{e_{5}}{\left|e_{5}\right|+\varepsilon} \\
& \dot{\hat{x}}_{6}=-\alpha_{2} e_{5}+\hat{x}_{2} \hat{x}_{4}\left(\frac{I_{x}-I_{y}}{I_{z}}\right)+\frac{1}{I_{z}} u_{4}-k_{2} \frac{e_{5}}{\left|e_{5}\right|+\varepsilon} \\
& \dot{\hat{x}}_{7}=-\alpha_{1} e_{7}+\hat{x}_{8}-k_{1} \frac{e_{7}}{\left|e_{7}\right|+\varepsilon} \\
& \dot{\hat{x}}_{8}=-\alpha_{2} e_{7}-g+\frac{c x_{1} c x_{3}}{m} u_{1}-k_{2} \frac{e_{7}}{\left|e_{7}\right|+\varepsilon} \\
& \dot{\hat{x}}_{9}=-\alpha_{1} e_{9}+\hat{x}_{10}-k_{1} \frac{e_{9}}{\left|e_{9}\right|+\varepsilon} \\
& \dot{\hat{x}}_{10}=-\alpha_{2} e_{9}+\frac{c x_{1} s x_{3} c x_{5}+s x_{1} s x_{5}}{m} u_{1}-k_{2} \frac{e_{9}}{\left|e_{9}\right|+\varepsilon} \\
& \dot{\hat{x}}_{11}=-\alpha_{1} e_{11}+\hat{x}_{12}-k_{1} \frac{e_{11}}{\left|e_{11}\right|+\varepsilon} \\
& \dot{\hat{x}}_{12}=-\alpha_{2} e_{11}+\frac{c x_{1} s x_{3} s x_{5}-s x_{1} c x_{5}}{m} u_{1}-k_{2} \frac{e_{11}}{\left|e_{11}\right|+\varepsilon}
\end{aligned}
$$

\subsubsection{Chattering elimination: sliding mode-based interval type-2 fuzzy observer}

Interval type-2 fuzzy logic system (IT2FLS) is introduced to eliminate chattering due to switching control of SMO. Generally, the architecture of IT2FLS consists of fuzzification, fuzzy inference and rule base, type reduction, and deffuzification as seen in Figure 5. This configuration is similar to type- 1 fuzzy logic systems except in the output processing block. In IT2FLS, the fuzzy output sets proceed to type reduction, then defuzzification to produce crisp outputs.

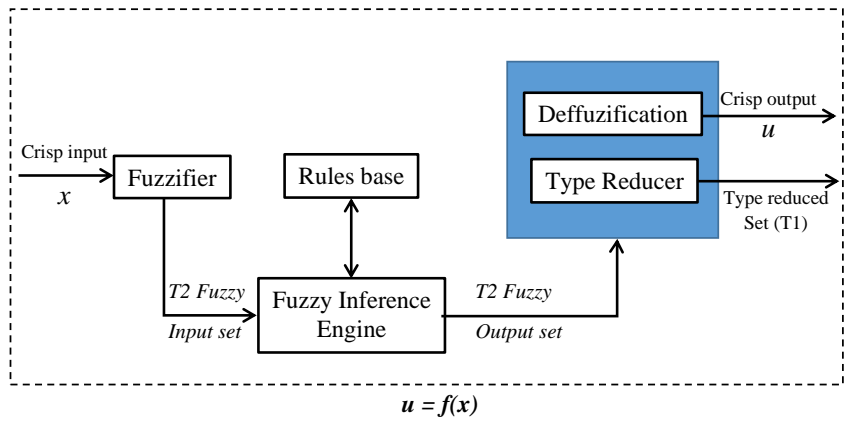

Figure 5. Architecture of IT2FLS

IT2FLS will play a role in replacing discontinuous term of SMO (31) to eliminate the chattering phenomenon. The membership function of fuzzy input is developed by the values of estimated states error $(e)$ as shown in Figure 6, while the fuzzy output is presented in Figure 7. Each of these has 7 membership functions, including: Negative Big (NB), Negative Medium (NM), Negative Small (NS), Zero (Z), Positive Small (PS), Positive Medium (PM), and Positive Big (PB) (Firdaus and Tokhi, 2015).

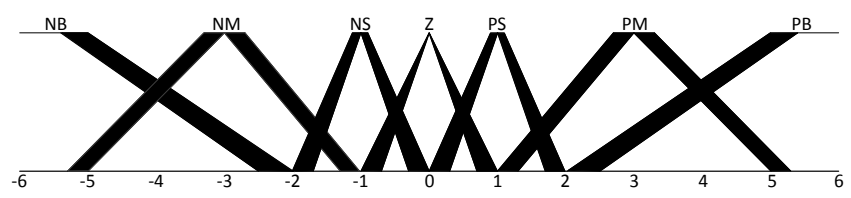

Figure 6. Estimation errors (e) MFs 


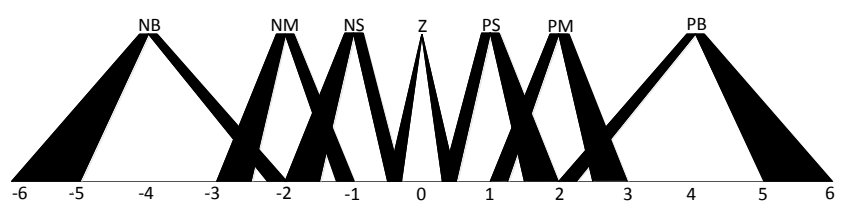

Figure 7. Fuzzy output MFs of SMC

Furthermore, considering the fuzzy input and output, the rules-base to generate the expected estimated states $(\hat{x})$ can be set up as in Table 1 .

Table 1. Fuzzy rules

\begin{tabular}{llllllll}
\hline & \multicolumn{6}{c}{$e$ (Error estimation) } \\
\cline { 2 - 7 } & NB & NM & NS & Z & PS & PM & PB \\
\hline $\boldsymbol{O u t}_{f 2}$ & NB & NM & NS & Z & PS & PM & PB \\
\hline
\end{tabular}

Hence, the IT2FSMO for the quadcopter model is presented as:

$$
\begin{aligned}
& \dot{\hat{x}}_{1}=-\alpha_{1} e_{1}+\hat{x}_{2}-k_{1} \boldsymbol{O u t}_{f 2}\left(e_{1}\right) \\
& \dot{\hat{x}}_{2}=-\alpha_{2} e_{1}+\hat{x}_{4} \hat{x}_{6}\left(\frac{I_{y}-I_{z}}{I_{x}}\right)+\frac{l}{I_{x}} u_{2}-k_{2} \boldsymbol{O u t}_{f 2}\left(e_{1}\right) \\
& \dot{\hat{x}}_{3}=-\alpha_{1} e_{3}+\hat{x}_{4}-k_{1} \boldsymbol{O u t}_{f 2}\left(e_{3}\right) \\
& \dot{\hat{x}}_{4}=-\alpha_{2} e_{3}+\hat{x}_{2} \hat{x}_{6}\left(\frac{I_{z}-I_{x}}{I_{y}}\right)+\frac{l}{I_{y}} u_{3}-k_{2} \boldsymbol{O u t}_{f 2}\left(e_{3}\right) \\
& \dot{\hat{x}}_{5}=-\alpha_{1} e_{5}+\hat{x}_{6}-k_{1} \boldsymbol{O u t}_{f 2}\left(e_{5}\right) \\
& \dot{\hat{x}}_{6}=-\alpha_{2} e_{5}+\hat{x}_{2} \hat{x}_{4}\left(\frac{I_{x}-I_{y}}{I_{z}}\right)+\frac{1}{I_{z}} u_{4}-k_{2} \boldsymbol{O} \boldsymbol{O u t _ { f 2 }}\left(e_{5}\right) \\
& \dot{\hat{x}}_{7}=-\alpha_{1} e_{7}+\hat{x}_{8}-k_{1} \boldsymbol{O u t}_{f 2}\left(e_{7}\right) \\
& \dot{\hat{x}}_{8}=-\alpha_{2} e_{7}-g+\frac{c x_{1} c x_{3}}{m} u_{1}-k_{2} \boldsymbol{O u t}_{f 2}\left(e_{7}\right) \\
& \dot{\hat{x}}_{9}=-\alpha_{1} e_{9}+\hat{x}_{10}-k_{1} \boldsymbol{O u t}_{f 2}\left(e_{9}\right) \\
& \dot{\hat{x}}_{10}=-\alpha_{2} e_{9}+\frac{c x_{1} s x_{3} c x_{5}+s x_{1} s x_{5}}{m} u_{1} k_{2} \boldsymbol{O u t} t_{f 2}\left(e_{9}\right) \\
& \dot{\hat{x}}_{11}=-\alpha_{1} e_{11}+\hat{x}_{12}-k_{1} \boldsymbol{O u t}_{f 2}\left(e_{11}\right) \\
& \dot{\hat{x}}_{12}=-\alpha_{2} e_{11}+\frac{c x_{1} s x_{3} s x_{5}-s x_{1} c x_{5}}{m} u_{1}-k_{2} \boldsymbol{O u t} t_{f 2}\left(e_{11}\right)
\end{aligned}
$$

where $\boldsymbol{O u t}_{f 2}$ represents type-2 fuzzy logic output.

\subsubsection{Chattering attenuation: super-twisting algorithm of sliding mode observer}

Considering the system as in equation (15), the supertwisting algorithm used for this class of systems has the following structure (Salgado et al., 2011)

$$
\begin{aligned}
& \dot{\hat{x}}_{1}=\hat{x}_{2}-k_{1} \sqrt{\left|e_{1}\right|} \frac{e_{1}}{\left|e_{1}\right|} \\
& \dot{\hat{x}}_{2}=f(x, t)+b(x, t) u(t)-k_{2} \frac{e_{1}}{\left|e_{1}\right|}
\end{aligned}
$$

In this structure, the gains $k_{i} i=1,2$ must be selected in such a way to ensure finite time convergence.

Hence, the STASMO for quadcopter UAVs can be defined as

$$
\begin{aligned}
& \dot{\hat{x}}_{1}=\hat{x}_{2}-k_{1} \sqrt{\left|e_{1}\right|} \frac{e_{1}}{\left|e_{1}\right|} \\
& \dot{\hat{x}}_{2}=\hat{x}_{4} \hat{x}_{6}\left(\frac{I_{y}-I_{z}}{I_{x}}\right)+\frac{l}{I_{x}} u_{2}-k_{2} \frac{e_{1}}{\left|e_{1}\right|} \\
& \dot{\hat{x}}_{3}=\hat{x}_{4}-k_{1} \sqrt{\left|e_{3}\right|} \frac{e_{3}}{\left|e_{3}\right|} \\
& \dot{\hat{x}}_{4}=\hat{x}_{2} \hat{x}_{6}\left(\frac{I_{z}-I_{x}}{I_{y}}\right)+\frac{l}{I_{y}} u_{3}-k_{2} \frac{e_{3}}{\left|e_{3}\right|} \\
& \dot{\hat{x}}_{5}=\hat{x}_{6}-k_{1} \sqrt{\left|e_{5}\right|} \frac{e_{5}}{\left|e_{5}\right|} \\
& \dot{\hat{x}}_{6}=\hat{x}_{2} \hat{x}_{4}\left(\frac{I_{x}-I_{y}}{I_{z}}\right)+\frac{1}{I_{z}} u_{4}-k_{2} \frac{e_{5}}{\left|e_{5}\right|} \\
& \dot{\hat{x}}_{7}=\hat{x}_{8}-k_{1} \sqrt{\left|e_{7}\right|} \frac{e_{7}}{\left|e_{7}\right|} \\
& \dot{\hat{x}}_{8}=-g+\frac{c x_{1} c x_{3}}{m} u_{1}-k_{2} \frac{e_{7}}{\left|e_{7}\right|} \\
& \dot{\hat{x}}_{9}=\hat{x}_{10}-k_{1} \sqrt{\left|e_{9}\right|} \frac{e_{9}}{\left|e_{9}\right|} \\
& \dot{\hat{x}}_{10}=\frac{c x_{1} s x_{3} c x_{5}+s x_{1} s x_{5}}{m} u_{1}-k_{2} \frac{e_{9}}{\left|e_{9}\right|} \\
& \dot{\hat{x}}_{11}=\hat{x}_{12}-k_{1} \sqrt{\left|e_{11}\right|} \frac{e_{11}}{\left|e_{11}\right|} \\
& \dot{\hat{x}}_{12}=\frac{c x_{1} s x_{3} s x_{5}-s x_{1} c x_{5}}{m} u_{1}-k_{2} \frac{e_{11}}{\left|e_{11}\right|}
\end{aligned}
$$

\subsubsection{Chattering attenuation: higher order sliding mode observer}

Second-order sliding modes cause the switching variables converge to zero in finite time when the relative degree of the variable equals two. In addition, the sliding modes can solve the chattering issue by employing continuous control if the relative degree is one. This may help to cancel harmful high-energy oscillations. Higher-order sliding modes (HOSMs) can handle the problem for arbitrary relative degrees. The implementation of the approach needs more information: usually it needs to calculate a number of sequential time derivatives of the switching variables.

General form of HOSMO is presented as (GonzalezHernandez et al., 2017, Skender et al., 2017)

$$
\begin{aligned}
& \dot{\hat{x}}_{1}=\hat{x}_{2}+k_{1}\left|e_{1}\right|^{\frac{2}{3}} \frac{e_{1}}{\left|e_{1}\right|} \\
& \dot{\hat{x}}_{2}=\hat{x}_{3}+k_{2}\left|e_{1}\right|^{\frac{1}{3}} \frac{e_{1}}{\left|e_{1}\right|}+f(x, t)+b(x, t) u(u) \\
& \dot{\hat{x}}_{3}=k_{3} \frac{e_{1}}{\left|e_{1}\right|}
\end{aligned}
$$

The dynamics of the estimated states errors are defined as,

$$
\begin{aligned}
& \dot{e}_{1}=e_{2}-k_{1}\left|e_{1}\right|^{\frac{2}{3}} \frac{e_{1}}{\left|e_{1}\right|} \\
& \dot{e}_{2}=-\hat{x}_{3}-k_{2}\left|e_{1}\right|^{\frac{1}{3}} \frac{e_{1}}{\left|e_{1}\right|}+\zeta \\
& \dot{e}_{3}=k_{3}\left|e_{1}\right|^{\frac{2}{3}} \frac{e_{1}}{\left|e_{1}\right|}
\end{aligned}
$$

Define a new variable of estimation error $e_{3}=\zeta-\hat{x}_{3}$, if the rate of the disturbance is restricted $|\dot{\zeta}|<\zeta^{+}$, then the dynamics of the estimated states error can be expressed as,

$$
\begin{aligned}
& \dot{e}_{1}=e_{2}-k_{1}\left|e_{1}\right|^{\frac{2}{3}} \frac{e_{1}}{\left|e_{1}\right|} \\
& \dot{e}_{2}=e_{3}-k_{2}\left|e_{1}\right|^{\frac{1}{3}} \frac{e_{1}}{\left|e_{1}\right|} \\
& \dot{e}_{3}=-k_{3}\left|e_{1}\right|^{\frac{2}{3}} \frac{e_{1}}{\left|e_{1}\right|}+\dot{\zeta}
\end{aligned}
$$


Before deriving HOSMO for quadcopter UAVs, it is important to redefine the states vector of such vehicle. The new states information are defined as

$$
\begin{aligned}
& x_{1}=\phi ; x_{4}=\theta ; x_{7}=\psi ; x_{10}=z ; x_{13}=x ; x_{16}=y \\
& x_{2}=\dot{\phi} ; x_{5}=\dot{\theta} ; x_{8}=\dot{\psi} ; x_{11}=\dot{z} ; x_{14}=\dot{x} ; x_{17}=\dot{y}
\end{aligned}
$$

Refers to the equation (40), the states estimation for quadcopter UAVs are obtained as follows

$$
\begin{aligned}
& \dot{\hat{x}}_{1}=\hat{x}_{2}+k_{1}\left|e_{1}\right|^{\frac{2}{3}} \frac{e_{1}}{\left|e_{1}\right|} \\
& \dot{\hat{x}}_{2}=\hat{x}_{3}+\hat{x}_{5} \hat{x}_{8}\left(\frac{I_{y}-I_{z}}{I_{x}}\right)+\frac{l}{I_{x}} u_{2}+k_{2}\left|e_{1}\right|^{\frac{1}{3}} \frac{e_{1}}{\left|e_{1}\right|} \\
& \dot{\hat{x}}_{3}=k_{3} \frac{e_{1}}{\left|e_{1}\right|} \\
& \dot{\hat{x}}_{4}=\hat{x}_{5}+k_{1}\left|e_{4}\right|^{\frac{2}{3}} \frac{e_{4}}{\left|e_{4}\right|} \\
& \dot{\hat{x}}_{5}=\hat{x}_{6}+\hat{x}_{2} \hat{x}_{8}\left(\frac{I_{z}-I_{x}}{I_{y}}\right)+\frac{l}{I_{y}} u_{3}+k_{2}\left|e_{4}\right|^{\frac{1}{3}} \frac{e_{4}}{\left|e_{4}\right|} \\
& \dot{\hat{x}}_{6}=k_{3} \frac{e_{4}}{\left|e_{4}\right|} \\
& \dot{\hat{x}}_{7}=\hat{x}_{8}+k_{1}\left|e_{7}\right|^{\frac{2}{3}} \frac{e_{7}}{\left|e_{7}\right|} \\
& \dot{\hat{x}}_{8}=\hat{x}_{9}+\hat{x}_{2} \hat{x}_{5}\left(\frac{I_{x}-I_{y}}{I_{z}}\right)+\frac{1}{I_{z}} u_{4}+k_{2}\left|e_{7}\right|^{\frac{1}{3}} \frac{e_{7}}{\left|e_{7}\right|} \\
& \dot{\hat{x}}_{9}=k_{3} \frac{e_{7}}{\left|e_{7}\right|} \\
& \dot{\hat{x}}_{10}=\hat{x}_{11}+k_{1}\left|e_{10}\right|^{\frac{2}{3}} \frac{e_{10}}{\left|e_{10}\right|} \\
& \dot{\hat{x}}_{11}=\hat{x}_{12}-g+\frac{c x_{1} c x_{4}}{m} u_{1}+k_{2}\left|e_{10}\right|^{\frac{1}{3}} \frac{e_{10}}{\left|e_{10}\right|} \\
& \dot{\hat{x}}_{12}=k_{3} \frac{e_{10}}{\left|e_{10}\right|} \\
& \dot{\hat{x}}_{13}=\hat{x}_{14}+k_{1}\left|e_{13}\right|^{\frac{2}{3}} \frac{e_{13}}{\left|e_{13}\right|} \\
& \dot{\hat{x}}_{14}=\hat{x}_{15}+\frac{c x_{1} s x_{4} c x_{7}+s x_{1} s x_{7}}{m} u_{1}+k_{2}\left|e_{13}\right|^{\frac{1}{3}} \frac{e_{13}}{\left|e_{13}\right|} \\
& \dot{\hat{x}}_{15}=k_{3} \frac{e_{13}}{\left|e_{13}\right|} \\
& \dot{\hat{x}}_{16}=\hat{x}_{17}+k_{1}\left|e_{16}\right|^{\frac{2}{3}} \frac{e_{16}}{\left|e_{16}\right|} \\
& \dot{\hat{x}}_{17}=\hat{x}_{18}+\frac{c x_{1} s x_{4} s x_{7}-s x_{1} c x_{7}}{m} u_{1}+k_{2}\left|e_{16}\right|^{\frac{1}{3}} \frac{e_{16}}{\left|e_{16}\right|} \\
& \dot{\hat{x}}_{18}=k_{3} \frac{e_{16}}{\left|e_{16}\right|}
\end{aligned}
$$

\section{Numerical simulation results}

In this sections a set of numerical simulations are exhibited to verify and demonstrate the performance of sliding mode control and observer approaches in controlling the quadcopter and estimating the unmeasured states of such vehicle. Comparative assessment of estimators is presented. Chattering phenomenon, estimation errors and time required to track true states are the factors will be evaluated to facilitate comparative studies of observers, while maximum overshoot, rise time, chattering issue, steadystate error, and control loop time are taken into account to evaluate controller performance relating to the use of several different observers. Disturbances and uncertainties such as white Gaussian noise, and parameters mismatch have also been incorporated into the simulation to demonstrate the robustness of the system.
The moment of inertia (Turnigy H.A.L frame), mass, and length of quadcopter arms are real parameters obtained by measurement. All quadcopter UAVs

\begin{tabular}{|c|c|c|c|c|c|c|c|c|c|}
\hline \multicolumn{10}{|c|}{ Observer } \\
\hline \multicolumn{2}{|c|}{ QuasisMO } & \multicolumn{2}{|c|}{ IT2FSMO } & \multicolumn{2}{|c|}{ STASMO } & \multicolumn{2}{|c|}{ HOSMO } & \multicolumn{2}{|c|}{ EKF } \\
\hline$\alpha_{1}$ & 85.8 & $\alpha_{1}$ & 85.8 & $k_{1}$ & 1.0 & $k_{1}$ & 6.0 & $R$ & 0.001 \\
\hline$\alpha_{2}$ & 7.2 & $\alpha_{2}$ & 7.2 & $k_{2}$ & 1.1 & $k_{2}$ & 11.0 & $Q$ & 0.1 \\
\hline$k_{1}$ & 0.1 & $k_{1}$ & 0.1 & & & $k_{3}$ & 6.0 & & \\
\hline$k_{2}$ & 2.0 & $k_{2}$ & 2.0 & & & & & & \\
\hline
\end{tabular}
parameters used in the simulation are shown in Table 2 , meanwhile the parameters of observers can be seen in Table 3. Selected weighting factors $(\varsigma)$ are 0.9 for $x$ and $y$ states, 0.688 for others. The simulation run with sampling $\left(t_{s}\right)$ time 0.01 seconds. In addition to those parameters, white Gaussian noise disturbance to measured states are selected to test the performance of the methods as well as parameters mismatch. The parameters mismatch is restricted to moment of inertia of the UAVs. The signal to noise ratio (SNR) introduced to measured states are $10 \mathrm{~dB}$ for roll, pitch, and yaw angle, and $37 \mathrm{~dB}$ for $x, y$, and $z$ movement, while parameters mismatch to be tested is $50 \%$. In addition, the highest parameter mismatch will be sought to determine the robustness limits of the controller and observers.

Table 2. Quadcopter parameters

\begin{tabular}{lccc}
\hline \multirow{2}{*}{ Variables } & \multicolumn{3}{c}{ Values } \\
\cline { 2 - 4 } & Observer model & Plant & Unit \\
\hline$m$ & 1.79 & 1.79 & $\mathrm{Kg}$ \\
$l$ & 0.29 & 0.29 & $\mathrm{~m}$ \\
$I_{x}$ & 0.02615 & 0.02615 & $\mathrm{Ns}^{2} / \mathrm{rad}$ \\
$I_{y}$ & 0.02735 & 0.02735 & $\mathrm{Ns}^{2} / \mathrm{rad}$ \\
$I_{z}$ & 0.04538 & 0.04538 & $\mathrm{Ns} / \mathrm{rad}$ \\
$k_{i}(i=1,2,3)$ & 0.37 & 0.37 & $\mathrm{Ns} / \mathrm{m}$ \\
$k_{j}(j=4,5,6)$ & 0.0005 & 0.0005 & $\mathrm{Ns} / \mathrm{m}$ \\
$g$ & 9.81 & 9.81 & $\mathrm{~m} / \mathrm{s}^{2}$ \\
\hline
\end{tabular}

Table 3. Observers parameters

There are two conditions to examine the performance of the control and observer methods, including: without noise and no parameters mismatch, and with noise and with parameters mismatch. The purpose of this approach is to highlight the ability of the controller and observers in dealing with several conditions.

In this numerical validation, four sliding modebased observer methods and extended Kalman filter are assessed. Suitable values for set-point weighting-based dynamic sliding mode control (SDISMC) are selected as,

$$
\begin{aligned}
& \lambda_{\phi}=10.0 \quad \lambda_{\theta}=10.0 \quad \lambda_{\psi}=15.0 \\
& \lambda_{x}=4.0 \quad \lambda_{y}=4.0 \quad \lambda_{z}=30.0 \\
& c_{\phi}=20.0 \quad c_{\theta}=20.0 \quad c_{\psi}=5.0 \\
& c_{x}=0.9 \quad c_{y}=0.9 \quad c_{z}=10.0 \\
& \lambda_{i \phi}=0.01 \quad \lambda_{i \theta}=0.01 \quad \lambda_{i \psi}=2.86 \\
& \lambda_{i x}=0.053 \lambda_{i y}=0.053 \lambda_{i z}=6.22 \\
& k_{\phi}=1.5 \quad k_{\theta}=1.5 \quad k_{\psi}=1.0 \\
& k_{x}=0.1 \quad k_{y}=0.1 \quad k_{z}=2.0 \\
& \mu_{\phi}=100.0 \mu_{\theta}=100.0 \mu_{\psi}=4.5 \\
& \mu_{x}=10,0 \quad \mu_{y}=10.0 \quad \mu_{z}=3.0
\end{aligned}
$$

\subsection{Without noise and no parameters mismatch}

For the first stage, noise-free condition and no parameters mismatch between plant and system model 
are used to evaluate the behaviour of the controller and observers. The simulation results obtained are presented in Figure 8 - 23.

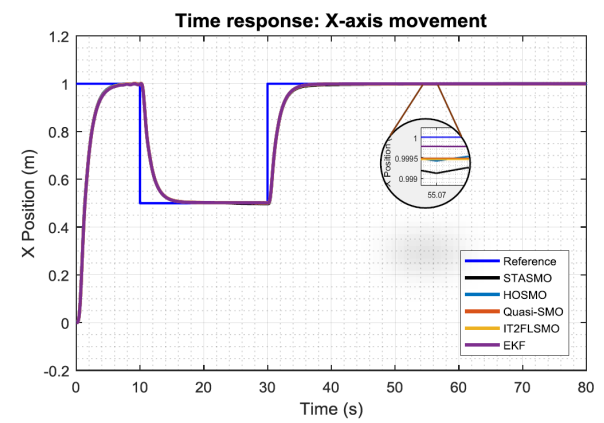

Figure 8. $x$-axis movement

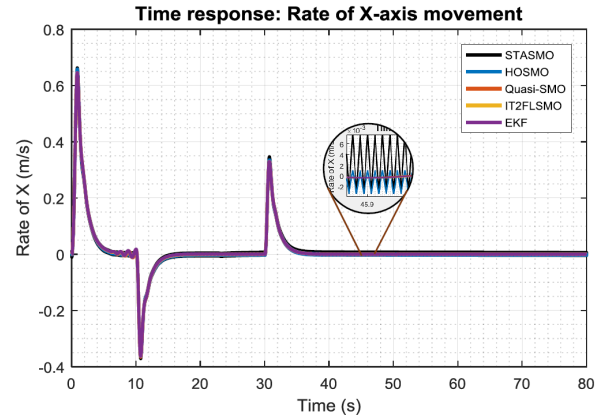

Figure 9. Rate of $x$-axis movement

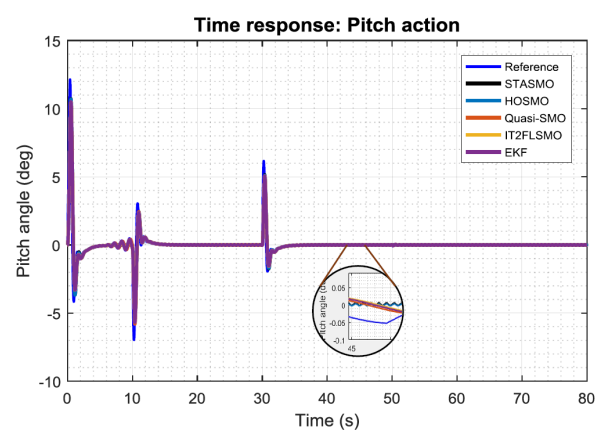

Figure 10. Pitch action

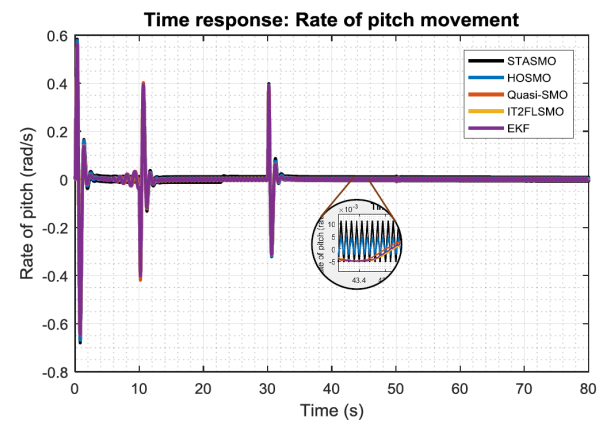

Figure 11. Rate of pitch movement

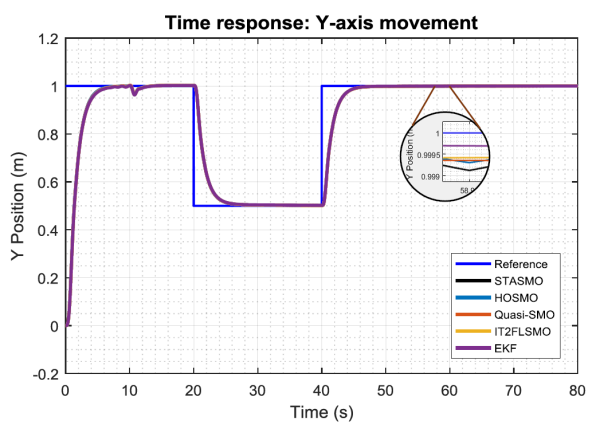

Figure 12. $y$-axis movement

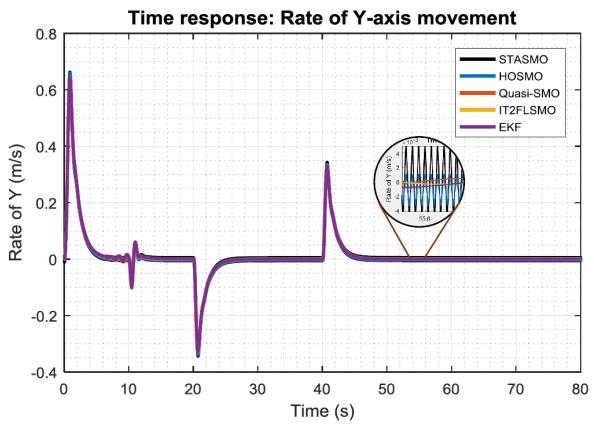

Figure 13. Rate of $y$-axis movement

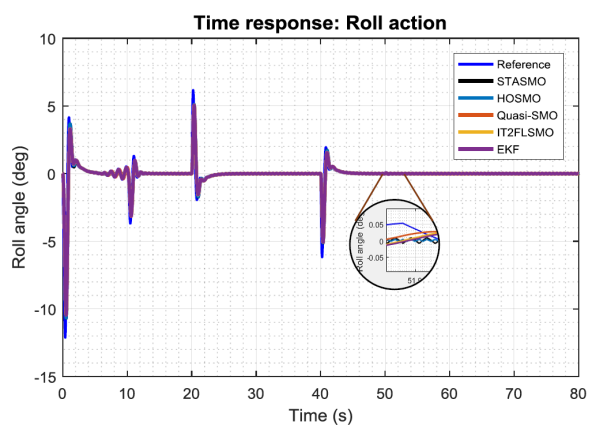

Figure 14. Roll action

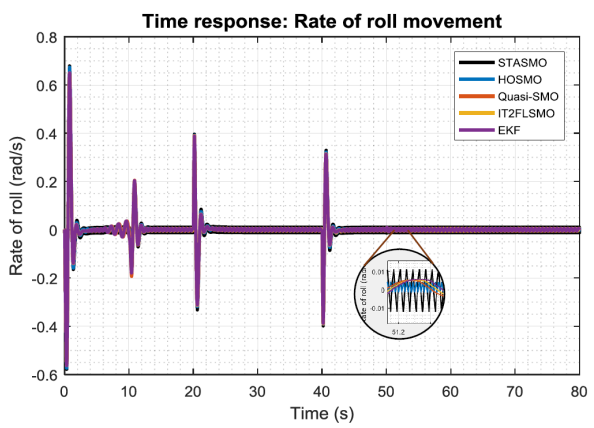

Figure 15. Rate of roll movement

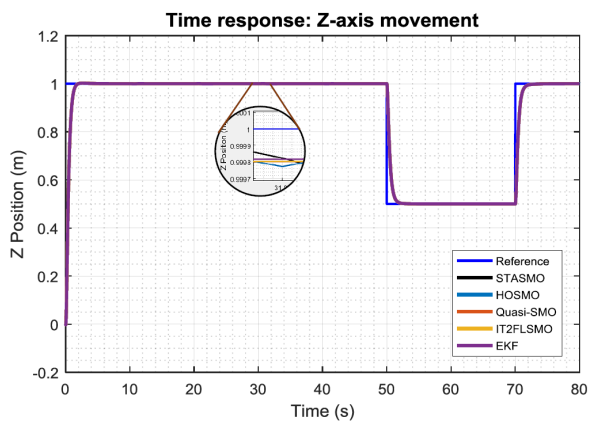

Figure 16. $z$-axis movement 


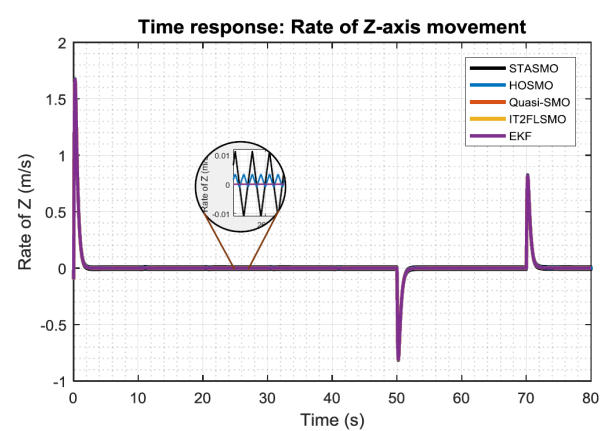

Figure 17. Rate of $z$-axis movement

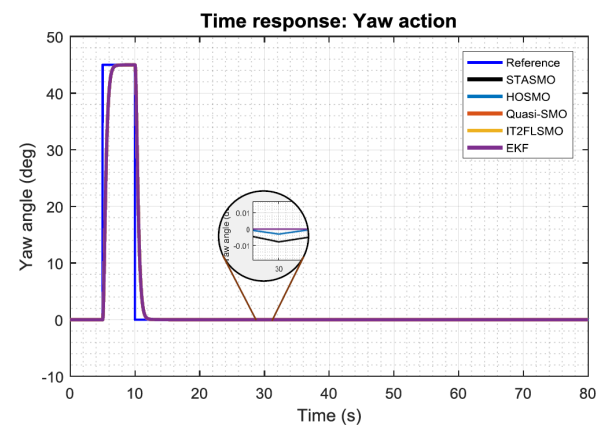

Figure 18. Yaw action

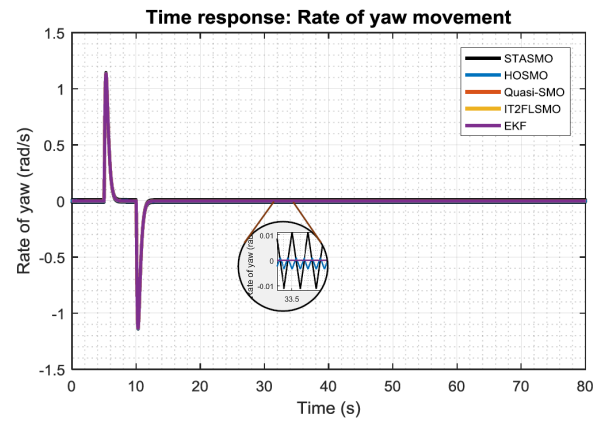

Figure 19. Rate of yaw movement

As noted, all observers, in general performed well in estimating unmeasured states of the quadcopter as can be seen in Figure 9, 11, 13, 15, 17, and 19. However, although the chattering issue was reduced significantly, switching function used in STASMO and HOSMO approaches is still the main cause of the slight oscillation in the estimated states.

Mean squared error (MSE) of estimated states in steady-state condition was calculated as presented in Table 4 to measure the quality of observers. IT2FSMO had smaller MSE in $x$, pitch and $z$ rate whereas QuasiSMO was better in estimating $y$ and yaw rate states. HOSMO was good in generating estimated state of roll rate. However, the MSE values between QuasiSMO and IT2FSMO were quite close so that both observers are considered to have similar performance in estimating unmeasured states.
Table 4. Mean squared states of estimated states

\begin{tabular}{lccc}
\hline \multirow{2}{*}{ Observer methods } & \multicolumn{3}{c}{ MSE steady-state error of } \\
\cline { 2 - 4 } & x rate & y rate & z rate \\
\hline QuasiSMO & $8.98791 \mathrm{E}-08$ & $9.11871 \mathrm{E}-08$ & $1.99643 \mathrm{E}-07$ \\
IT2FLSMO & $8.75774 \mathrm{E}-08$ & $1.08689 \mathrm{E}-07$ & $1.97097 \mathrm{E}-07$ \\
STASMO & $3.38737 \mathrm{E}-05$ & $3.03218 \mathrm{E}-05$ & $6.46195 \mathrm{E}-05$ \\
HOSMO & $6.29399 \mathrm{E}-06$ & $6.27793 \mathrm{E}-06$ & $6.75966 \mathrm{E}-06$ \\
EKF & $9.50554 \mathrm{E}-08$ & $2.97137 \mathrm{E}-07$ & $2.02128 \mathrm{E}-07$ \\
\hline & pitch rate & roll rate & yaw rate \\
\hline QuasiSMO & $1.33916 \mathrm{E}-05$ & $1.34791 \mathrm{E}-05$ & $8.19673 \mathrm{E}-10$ \\
IT2FLSMO & $1.33371 \mathrm{E}-05$ & $1.44075 \mathrm{E}-05$ & $8.19678 \mathrm{E}-10$ \\
STASMO & $7.40598 \mathrm{E}-05$ & $7.25747 \mathrm{E}-05$ & $6.28488 \mathrm{E}-05$ \\
HOSMO & $1.36294 \mathrm{E}-05$ & $9.43137 \mathrm{E}-06$ & $6.61028 \mathrm{E}-06$ \\
EKF & $1.35298 \mathrm{E}-05$ & $1.38534 \mathrm{E}-05$ & $8.21467 \mathrm{E}-10$ \\
\hline
\end{tabular}

In addition to estimating unmeasured states, each observer showed different performance in tracking true states. The ability of the observers in generating estimation of true states is presented in Table 5. In the case of without noise and no parameters mismatch, the QuasiSMO and IT2FSMO outperformed other methods by showing smaller MSE for pitch, roll, yaw, and $z$ states, whereas EKF was better in $x$ and $y$ than others.

Table 5. Mean squared errors of true states tracking

\begin{tabular}{lccc}
\hline \multirow{2}{*}{ Observer method } & \multicolumn{3}{c}{ MSE state estimation of } \\
\cline { 2 - 4 } & $\mathbf{x}$ & $\mathbf{y}$ & $\mathbf{z}$ \\
\hline QuasiSMO & $3.11324 \mathrm{E}-10$ & $3.13195 \mathrm{E}-10$ & $3.39063 \mathrm{E}-12$ \\
IT2FLSMO & $3.12907 \mathrm{E}-10$ & $3.15066 \mathrm{E}-10$ & $3.40674 \mathrm{E}-12$ \\
STASMO & $5.31837 \mathrm{E}-09$ & $4.90389 \mathrm{E}-09$ & $9.20483 \mathrm{E}-09$ \\
HOSMO & $2.98112 \mathrm{E}-09$ & $2.97376 \mathrm{E}-09$ & $2.97571 \mathrm{E}-09$ \\
EKF & $7.76156 \mathrm{E}-11$ & $8.68866 \mathrm{E}-11$ & $4.30985 \mathrm{E}-09$ \\
\hline & pitch & roll & yaw \\
\hline QuasiSMO & $2.35233 \mathrm{E}-12$ & $2.44451 \mathrm{E}-13$ & $6.36673 \mathrm{E}-15$ \\
IT2FLSMO & $3.96453 \mathrm{E}-12$ & $7.87942 \mathrm{E}-14$ & $6.41306 \mathrm{E}-15$ \\
STASMO & $6.70786 \mathrm{E}-09$ & $9.19166 \mathrm{E}-09$ & $9.21405 \mathrm{E}-09$ \\
HOSMO & $2.97349 \mathrm{E}-09$ & $2.97351 \mathrm{E}-09$ & $2.97384 \mathrm{E}-09$ \\
EKF & $1.64104 \mathrm{E}-09$ & $1.67363 \mathrm{E}-09$ & $1.76539 \mathrm{E}-09$ \\
\hline
\end{tabular}

The performance of observers was evaluated also by time required to estimate unknown states and to track true states since this parameter plays a critical role in real-time applications. It was noted in previous section that each observer had different numerical methods to observe the unknown states. These differences will result in varying amount of time required to estimate unknown states and to track true states as presented in Table 6 .

Table 6. Time required to estimate unmeasured states

\begin{tabular}{cccccc}
\hline & QSMO & IT2SMO & STASMO & HOSMO & EKF \\
\hline$t(\mathrm{~ms})$ & 0.04125 & 4.02375 & 0.05925 & 0.0755 & 0.1425 \\
\hline
\end{tabular}

Although IT2FSMO has good performance in estimating unknown states, this method needs more time to track measured states and estimate second order states than other methods. Type reduction method in type-2 fuzzy logic is one of the issues that cause such delay. Type reduction is still a fascinating topic to be researched in type-2 fuzzy logic field so that it can be used in a wide range of real-time applications. As noted, among the observers, QuasiSMO was the fastest estimator followed by STASMO, HOSMO and EKF.

From a control view point, the chattering issue of estimated states following the use STASMO and 
HOSMO are not major problem as SDISMC could control altitude and attitude of quadcopter UAV quite smoothly. It can be seen in Figure 8 - 19 with the good performance of the controller in tracking references.

The SDISMC showed good performance in controlling the quadcopter with various observers. It can be seen in the results presented that there was no overshoot with the controller in response to a step input for all observers methods with similar rise time. However, significant oscillations were seen in the control inputs of roll and pitch movements using STASMO and HOSMO as can be seen in Figure 21 and 22, whereas QuasiSMO, IT2FSMO and EKF could give smoother control signals.

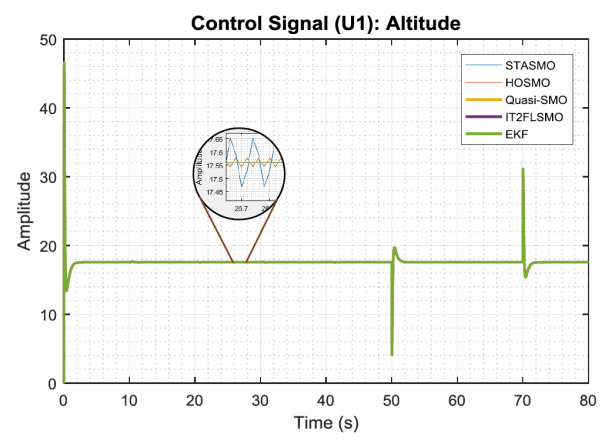

Figure 20. Control signal $u_{1}$

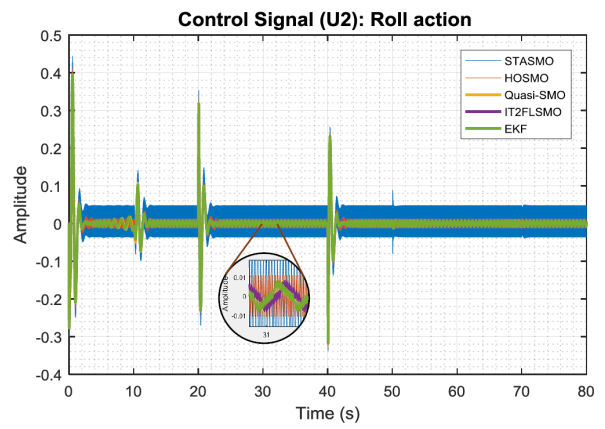

Figure 21. Control signal $u_{2}$

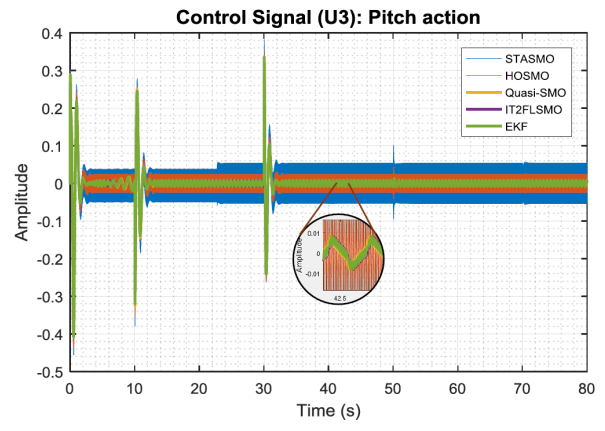

Figure 22. Control signal $u_{3}$

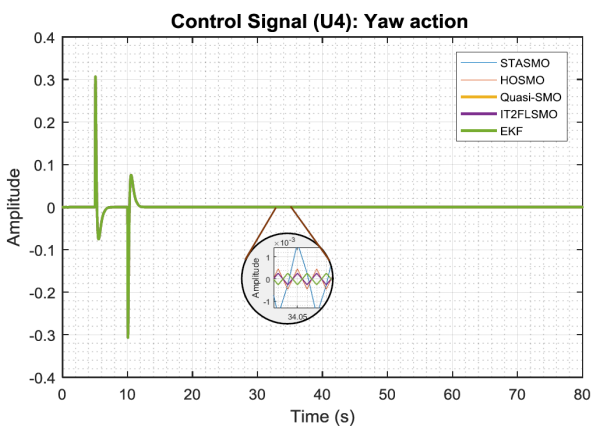

Figure 23. Control signal $u_{4}$

Another factor of consideration to evaluate the controller performance with the observers is steadystate error. Table 7 shows mean squared error of output states of the quadcopter UAV in steady-state condition. As noted, all observers show similar performance in improving controller to reduce steady-states error in almost all states except in yaw state where STASMO and HOSMO show higher MSE significantly than others.

Table 7. Mean squared errors of steady-states errors

\begin{tabular}{lrrr}
\hline \multirow{2}{*}{ Observer methods s } & \multicolumn{3}{c}{ MSE steady-state error of } \\
\cline { 2 - 4 } & $\mathbf{x}$ & $\mathbf{y}$ & $\mathbf{z}$ \\
\hline QuasiSMO & $3.32422 \mathrm{E}-07$ & $3.5677 \mathrm{E}-07$ & $1.87105 \mathrm{E}-08$ \\
IT2FLSMO & $3.43706 \mathrm{E}-07$ & $3.54602 \mathrm{E}-07$ & $1.88812 \mathrm{E}-08$ \\
STASMO & $2.51545 \mathrm{E}-06$ & $5.99312 \mathrm{E}-07$ & $2.87283 \mathrm{E}-08$ \\
HOSMO & $3.76194 \mathrm{E}-07$ & $3.53125 \mathrm{E}-07$ & $2.15704 \mathrm{E}-08$ \\
EKF & $8.713 \mathrm{E}-08$ & $1.06684 \mathrm{E}-07$ & $1.91677 \mathrm{E}-08$ \\
\hline & pitch & roll & yaw \\
\hline QuasiSMO & $4.04871 \mathrm{E}-07$ & $4.07073 \mathrm{E}-07$ & $1.88658 \mathrm{E}-13$ \\
IT2FLSMO & $3.77827 \mathrm{E}-07$ & $6.28935 \mathrm{E}-07$ & $1.86451 \mathrm{E}-13$ \\
STASMO & $3.68175 \mathrm{E}-07$ & $3.76505 \mathrm{E}-07$ & $9.47006 \mathrm{E}-09$ \\
HOSMO & $3.64405 \mathrm{E}-07$ & $3.69027 \mathrm{E}-07$ & $2.87142 \mathrm{E}-09$ \\
EKF & $4.45222 \mathrm{E}-07$ & $6.52378 \mathrm{E}-07$ & $3.55165 \mathrm{E}-13$ \\
\hline
\end{tabular}

In summary, in case of no noise and no parameters mismatch, SDISMC shows good performance in controlling the quadcopter UAV with no overshoot response, small rise time, very small chattering, and small steady-state errors. The set-point weighting term worked well in eliminating overshoot during integral term was being employed. In addition, the controller has fast computational time which is feasible for realtime application. Among the observers, QuasiSMO method outperforms other methods by showing smooth estimated states, smaller states estimation and true states tracking errors, and faster in computational time. Therefore, the observer has influenced and improved the overall performance of the controller.

\subsection{With noise and with parameters mismatch}

At this stage, the performance of control system and observers was tested by introducing noise and uncertainty at the same time into the quadcopter system. Robustness of the approaches will play a vital role in dealing with such disturbances. The simulation results obtained are shown in Figure 24 - 39. 


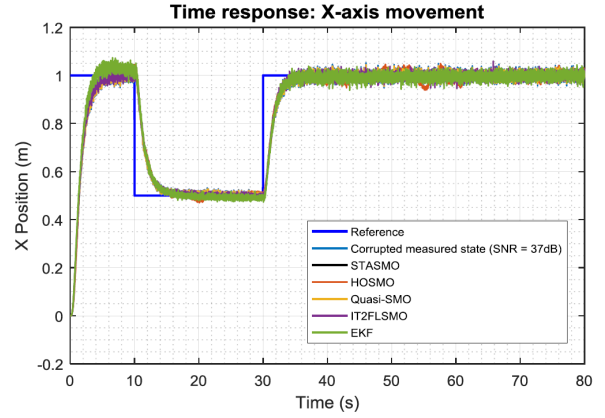

Figure 24. $x$-axis movement

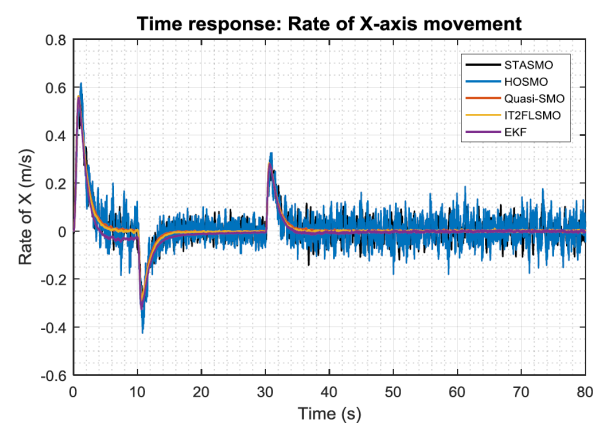

Figure 25. Rate of $x$-axis movement

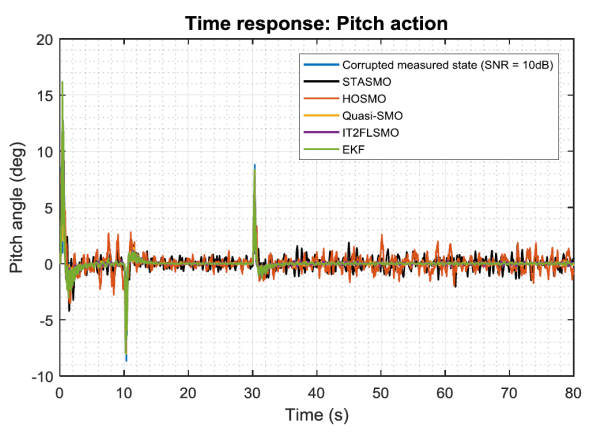

Figure 26. Pitch action

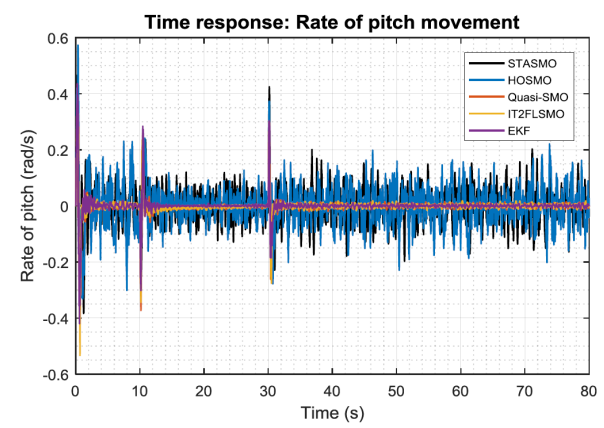

Figure 27. Rate of pitch movement

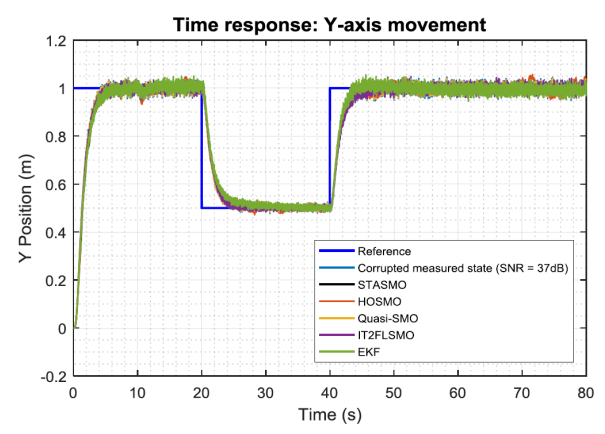

Figure 28. $y$-axis movement

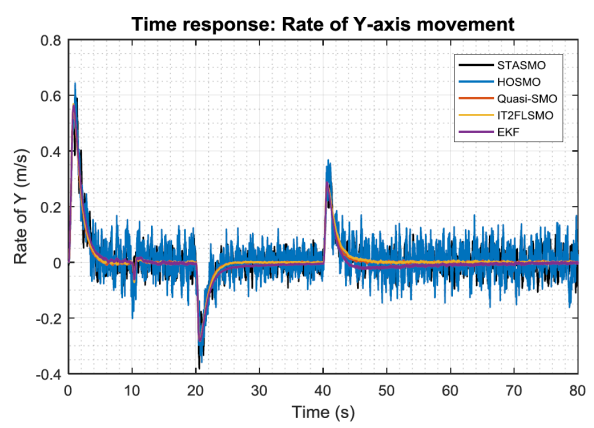

Figure 29. Rate of $y$-axis movement

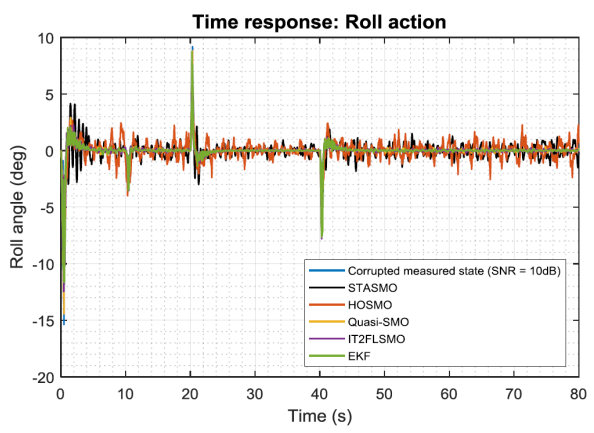

Figure 30. Roll action

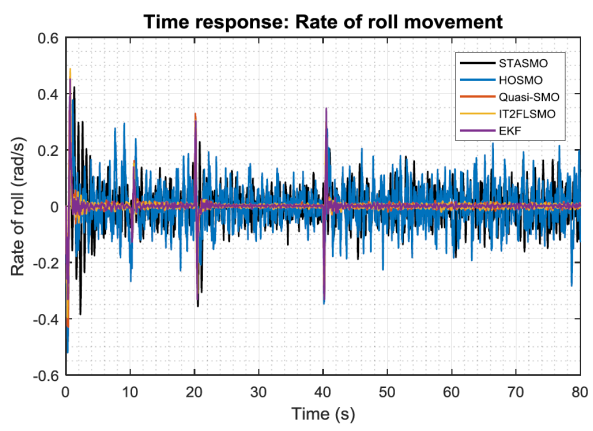

Figure 31. Rate of roll movement

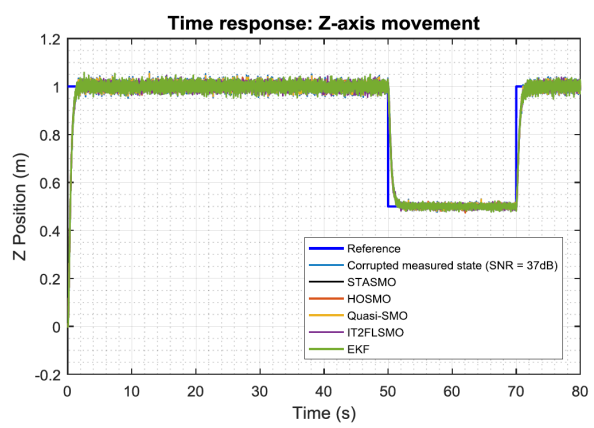

Figure 32. $z$-axis movement

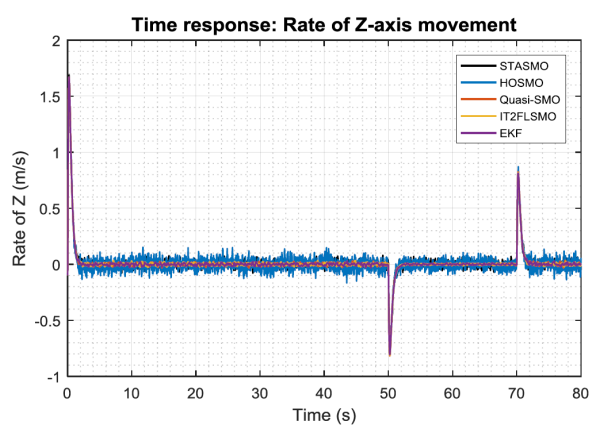

Figure 33. Rate of $z$-axis movement 


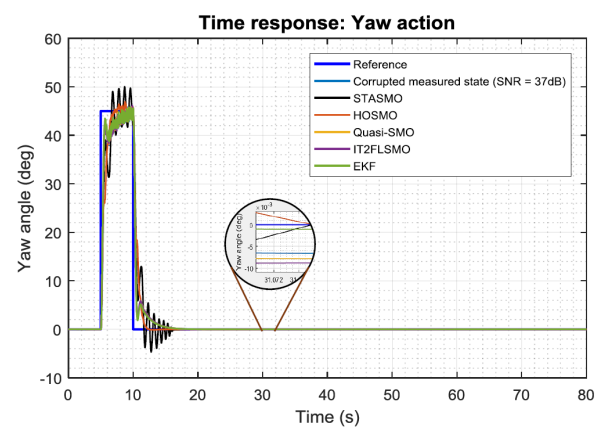

Figure 34. Yaw action

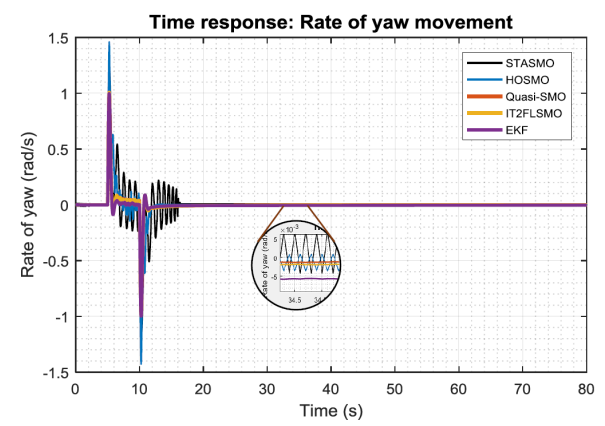

Figure 35. Rate of yaw movement

The simulation results presented in Figure 25, 27, 29, 31, 33, and 35 show that despite the presence of high power noise and high uncertainty, the effect of such disturbances were reduced sufficiently by QuasiSMO, IT2FSMO, and EKF so that second order states were generated quite smoothly. In other words, noise disturbance on the output states as well as parameter mismatch do not affect significantly the performance of the observers. In contrast, corrupted output states influenced results due to STASMO and HOSMO significantly, especially in estimating second order state of pitch, roll, and yaw as shown in the figures. The noise on the output states was amplified high enough due to the effect of switching functions in the methods. In addition, STASMO could not deal with parameters mismatch well, and this is shown with high amplitude oscillation in the estimation of yaw rate when the reference changes.

To evaluate the performance of observer numerically, MSE of estimated states in steady-state condition was calculated as presented in Table 8. Noise disturbance and uncertainty are inevitable conditions in quadcopter UAVs system which might disturb the performance of observers. However, as noted, the QuasiSMO, IT2FSMO, and EKF always maintain its performance in estimating unmeasured states. The estimators especially QuasiSMO constantly showed smaller MSE than STASMO and HOSMO despite being in a bad condition.
Table 8. Mean squared errors of estimated states

\begin{tabular}{lccr}
\hline \multirow{2}{*}{ Observer methods } & \multicolumn{3}{c}{ MSE steady-state error of } \\
\cline { 2 - 4 } & x rate & y rate & \multicolumn{1}{c}{ z rate } \\
\hline QuasiSMO & $4.71813 \mathrm{E}-06$ & $5.67621 \mathrm{E}-06$ & $8.31377 \mathrm{E}-05$ \\
IT2FLSMO & $4.78787 \mathrm{E}-06$ & $5.0615 \mathrm{E}-06$ & $8.25333 \mathrm{E}-05$ \\
STASMO & 0.001408557 & 0.001360913 & 0.000758379 \\
HOSMO & 0.002667377 & 0.002849846 & 0.001901026 \\
EKF & $1.54249 \mathrm{E}-05$ & $8.22583 \mathrm{E}-05$ & $8.2426 \mathrm{E}-05$ \\
\hline & pitch rate & roll rate & yaw rate \\
\hline QuasiSMO & $3.22091 \mathrm{E}-05$ & $3.87957 \mathrm{E}-05$ & $3.42127 \mathrm{E}-06$ \\
IT2FLSMO & $3.45078 \mathrm{E}-05$ & $3.24607 \mathrm{E}-05$ & $4.6615 \mathrm{E}-06$ \\
STASMO & 0.003987265 & 0.003837892 & $3.28468 \mathrm{E}-05$ \\
HOSMO & 0.004578211 & 0.005344991 & $6.56119 \mathrm{E}-06$ \\
EKF & $1.54136 \mathrm{E}-05$ & $1.85355 \mathrm{E}-05$ & $2.43512 \mathrm{E}-05$ \\
\hline
\end{tabular}

In the present of noise and parameter mismatch, the true states tracking errors look similar for all observer method. However, referring to computed MSE values as presented in Table 9, show that each observer technique exhibited different performance in tracking the true states. Although not able to estimate second order states as good as QuasiSMO, IT2FSMO and EKF; STASMO and HOSMO showed better performance in tracking the true states. It is noticeable in Table 9 that STAMSO and HOSMO had smaller MSE slightly than other approaches in tracking the measured states of $x, y$, yaw and $z$, while QuasiSMO and IT2FSMO showed better tracking of pitch and roll states. However, STASMO and HOSMO did not perform as well as other approaches in estimating second order states so the overall control performance would be affected.

Table 9. Mean squared errors of true states tracking

\begin{tabular}{lcrc}
\hline \multirow{2}{*}{ Observer method } & \multicolumn{3}{c}{ MSE state estimation of } \\
\cline { 2 - 4 } & $\mathbf{x}$ & $\mathbf{y}$ & $\mathbf{z}$ \\
\hline QuasiSMO & 0.000277102 & 0.000278138 & 0.000285373 \\
IT2FLSMO & 0.000276616 & 0.000271504 & 0.000286913 \\
STASMO & 0.000172541 & 0.000173697 & 0.000177637 \\
HOSMO & 0.000189739 & 0.000182192 & 0.000192498 \\
EKF & 0.000324588 & 0.000299838 & 0.000325291 \\
\hline & pitch & roll & yaw \\
\hline QuasiSMO & $2.31257 \mathrm{E}-05$ & $4.0883 \mathrm{E}-05$ & $1.19431 \mathrm{E}-05$ \\
IT2FLSMO & $2.86099 \mathrm{E}-05$ & $3.22704 \mathrm{E}-05$ & $1.18428 \mathrm{E}-05$ \\
STASMO & $7.25988 \mathrm{E}-05$ & 0.00010442 & 0.000445377 \\
HOSMO & $4.28961 \mathrm{E}-05$ & $3.85983 \mathrm{E}-05$ & $1.17318 \mathrm{E}-05$ \\
EKF & $3.9194 \mathrm{E}-05$ & $3.50203 \mathrm{E}-05$ & $1.37551 \mathrm{E}-05$ \\
\hline
\end{tabular}

The presence of noise and parameters mismatch is expected to affect the performance of the controller. However, the effect of those unforeseen circumstances on the system depend on management and selection of control and observer types. Figure 24, 26, 28, 30, 32, and 34 show that the use of all type of observer approaches did not have much effect on the control performance in controlling output states except yaw. As noted, the controller responds step input reference quite well by tracking the references quite smoothly. However, in the case of yaw state, oscillation occurs obviously around $\pm 5.0^{\circ}$ when STASMO was applied.

The noise effect was still noticeable clearly around references and control inputs as seen in Figure 36 - 39. However, the noise influenced control input considered as quite low by showing small amplitude of oscillation in the signals. The effects of noise still occur due to 
trade-off of choosing parameters for the observers. On the one hand, increasing or decreasing observer gains will speed up tracking true value, yet may potentially increase sensitivity to noise measurement and vice versa. In this research, the speed of tracking measured states is more considered (without neglecting noise effect) to make the controller more responsive.

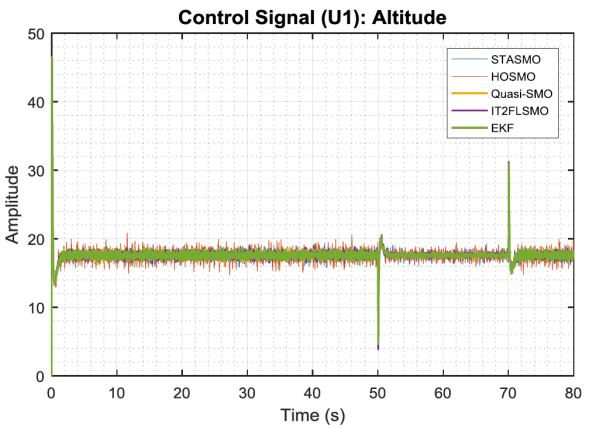

Figure 36. Control signal $u_{1}$

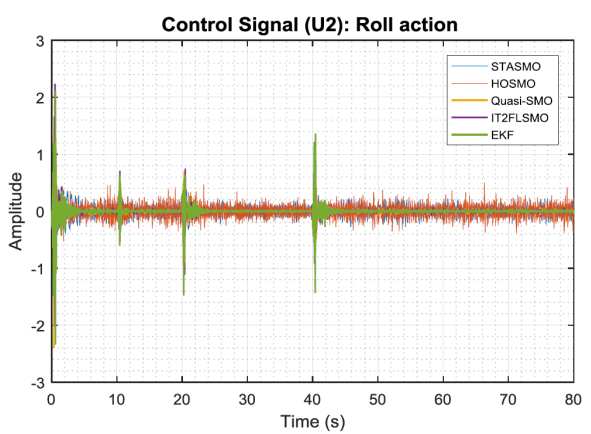

Figure 37. Control signal $u_{2}$

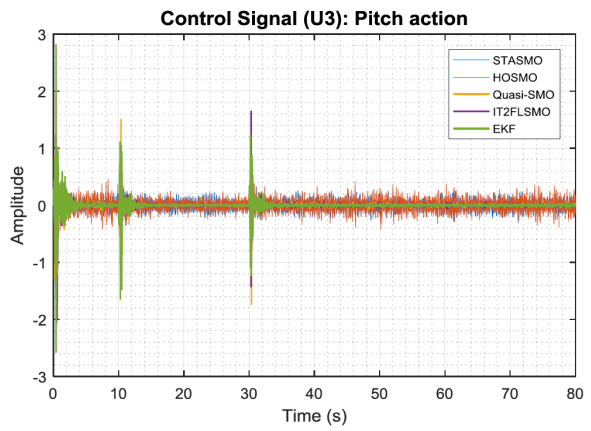

Figure 38. Control signal $u_{3}$

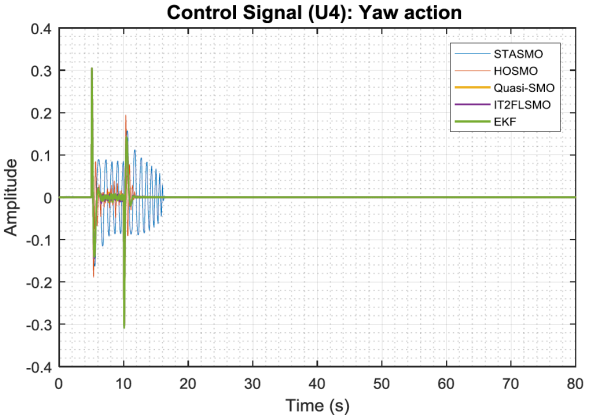

Figure 39. Control signal $u_{4}$

Furthermore, steady-states error is considered also to evaluate the robustness of SDISMC and observers in dealing with noise disturbance and parameter mismatch. The results in Table 10, show that, generally steadystates errors are considered small. It means that even though noise and parameters mismatch have put the quadcopter system in bad condition, the observers have influenced control system to stabilize quadcopter and reduce steady-state error quite well. In this case, STASMO observer method performs better than those of four estimators by showing smaller steady-state error in almost all states computed by MSE method.

Table 10. Mean squared errors of steady-states errors

\begin{tabular}{lcrr}
\hline \multirow{2}{*}{ Observer methods } & \multicolumn{3}{c}{ MSE steady-state error of } \\
\cline { 2 - 4 } & $\mathbf{x}$ & $\mathbf{y}$ & \multicolumn{1}{c}{$\mathbf{z}$} \\
\hline QuasiSMO & 0.000154099 & 0.000153519 & 0.000154979 \\
IT2FLSMO & 0.000164337 & 0.000153464 & 0.00015562 \\
STASMO & $7.92722 \mathrm{E}-05$ & $7.20529 \mathrm{E}-05$ & $3.46551 \mathrm{E}-05$ \\
HOSMO & 0.000412268 & 0.000420688 & $9.26384 \mathrm{E}-05$ \\
EKF & 0.000235152 & 0.000227582 & 0.000205899 \\
\hline & pitch & roll & yaw \\
\hline QuasiSMO & 0.00014667 & 0.000140496 & $1.00158 \mathrm{E}-08$ \\
IT2FLSMO & 0.00014533 & 0.000141111 & $8.50916 \mathrm{E}-09$ \\
STASMO & $8.08244 \mathrm{E}-05$ & $7.86021 \mathrm{E}-05$ & $5.89205 \mathrm{E}-09$ \\
HOSMO & 0.000275643 & 0.000302138 & $2.84122 \mathrm{E}-09$ \\
EKF & 0.000143496 & 0.000140625 & $1.92223 \mathrm{E}-09$ \\
\hline
\end{tabular}

In summary, in the presence of noise and parameter mismatch, SDISMC maintains good performance in controlling the dynamics of quadcopter with no response overshoot except for yaw action when employing STASMO, considered small rise time, free from chattering when using QuasiSMO, IT2FSMO and EKF observer method, small steady-states errors, and fast computational time. However, fluctuated signals randomly on the references still appear due to noise disturbance. From observer viewpoint, regarding true state tracking and steady-states errors, STASMO outperforms other methods by showing small MSE values. However, QuasiSMO, IT2FSMO, EKF perform better than others in reducing noise effect on state estimation process and tracking reference. It can be seen by smoother estimated states generated, and no oscillation issue in tracking reference.

\subsection{Summary}

From the simulation results obtained can be summarized that QuasiSMO, IT2FSMO, and EKF generated smoother estimated states for every conditions than STASMO and HOSMO. MSE estimated states in steadystate conditions was evaluated also to verify observers performance. In free of noise condition, QuasiSMO and IT2FSMO outperformed others by exhibiting smaller MSE in all states. Furthermore, in the presence of noise and parameters mismatch, QuasiSMO and IT2FSMO had smaller MSE for second order states of $x, y$, and yaw than other observers; while EKF was better than those of four estimators for roll and pitch rates.

The MSE of true states tracking and noise rejection were considered to evaluating the performance of observers. In the ideal condition, QuasiSMO dominated smaller MSE for all states, while STASMO and HOSMO had good performance by showing small MSE for most of states in the presence of noise and uncertainty. However, in terms of noise rejection, QuasiSMO, IT2FSMO and 
EKF had more capability in rejecting noise effect in estimating process than STASMO and HOSMO by exhibiting small random fluctuation in all estimated states. Actually, reducing or rejecting noise effect and fast tracking are trade-off for observer methods. On the one hand, increasing or decreasing estimator gains will speed up tracking true value, yet may potentially increase sensitivity to noise measurement and vice versa.

Each observer demonstrated different achievement in contributing to improving control system performance. In the use of QuasiSMO, IT2FSMO and EKF, the control system performed good performance by exhibiting no overshoot for all output states. Furthermore, in terms of steady-state errors, MSE was calculated and compared. For noise-free condition, no observer method dominated better performance. Nevertheless, in the appearance of noise, STASMO was dominating by showing smaller MSE in almost all states.

Time required to process control and observer algorithm is playing a important role in designing responsive control system. Hence, computational time of observers algorithm was measured as presented in the Table 6 . From the table it is noticeable clearly that QuasiSMO algorithm has faster time consumed than others around $0.04125 \mathrm{~ms}$, whereas IT2FSMO is the slowest one around $4.02375 \mathrm{~ms}$. Meanwhile, the time demand to process the sequence of the controller method is quite fast around $0.1 \mathrm{~ms}$. It means that the control method is feasible for experimental validation.

Chattering which is the main issue in SMCs, was reduced significantly by SDISMC so that what ever estimators were used, all estimated states were generated quite smoothly. In addition, time responses including rise time, overshoot, and steady-state error were evaluated. In most states and various uses of observer, the controller responded step inputs with similar rise time and no overshoot occurs except only in yaw state when STASMO and HOSMO approaches were employed in the condition of noise disturbance. In other words that set-point weighting term was successfully to reduce overshoot occurrence significantly because of employing integral function while keeping steady-state errors in small values.

Lastly, for further robustness testing in dealing with uncertainty, a percentage of increasing parameters mismatch was evaluated subject to noise disturbance. In this assessment, the robustness of the observer methods was shown by the stability of the system in responding step input references regarding the increase of parameter mismatch value. Therefore, the maximum values of parameter uncertainty percentage were obtained as presented in the Table 11. From the table, it can be seen clearly that the use QuasiSMO, IT2FSMO, and EKF outperformed other estimator approaches in dealing with such uncertainty.
Table 11. Maximum parameters mismatch subject to noise disturbance

\begin{tabular}{ccccc}
\hline \multicolumn{5}{c}{ Maximum parameters mismatch (\%) } \\
\hline QuasiSMO & IT2FSMO & STASMO & HOSMO & EKF \\
\hline 89.0 & 89.0 & 52.0 & 64.0 & 90.2 \\
\hline
\end{tabular}

\section{Conclusion}

After performing a series of performance assessments for control system and observer methods and considering the results obtained, it can be concluded that generally, set-point weighting-based dynamic integral sliding mode control worked well to control attitude and altitude of quadcopter UAVs in various conditions including noise disturbance and high uncertainty by showing good achievement in every predefined performance criteria. Meanwhile, although quasi sliding mode observer is a conventional method, the observer has a promising performance in estimating unmeasured states by maintaining good results in every determined categories, and more contribution than other approaches to improving the performance of the proposed control system. In addition, the method has faster processing time than other approaches which is crucial for plants that require highly responsive dynamic control system. Therefore, the combination of the methods is considered to be evaluated experimentally.

\section{Acknowledgement}

This work was supported by Indonesia Endowment Fund for Education, Ministry of Finance Republic of Indonesia, and Politeknik Negeri Batam, Indonesia.

\section{References}

Astolfi, D. and Marconi, L. (2015), 'A high-gain nonlinear observer with limited gain power', IEEE Transactions on Automatic Control 60(11), 30593064 .

Boiko, I. and Chehadeh, M. (2018), 'Sliding mode differentiator/observer for quadcopter velocity estimation through sensor fusion', International Journal of Control $\mathbf{0}(0), 1-8$.

Bouadi, H. and Tadjine, M. (2007), 'Nonlinear observer design and sliding mode control of four rotors helicopter', International Journal of Aerospace and Mechanical Engineering 1(7), 329-334.

Cen, Z., Noura, H., Susilo, T. B. and Younes, Y. A. (2014), 'Robust fault diagnosis for quadrotor uavs using adaptive thau observer', J. Intell. Robotics Syst. 73(1-4), 573-588. 
Chen, F. and Dunnigan, M. W. (2002), 'Comparative study of a sliding-mode observer and kalman filters for full state estimation in an induction machine', IEE Proceedings - Electric Power Applications 149(1), 5364.

Elamine, L., Meguenni, K., Youssouf, M. and Litim, M. (2013), 'Nonlinear observer and backstepping control of quadrotor unmanned aerial vehicle', International Review of Automatic Control (IREACO) 6(5).

Elkholy, H. (2014), Dynamic Modeling and Control of a Quadrotor Using Linear and Nonlinear Approaches, Thesis, American University in Cairo. School of Engineering Interdisciplinary Program, Egypt.

Firdaus, A. R. and Rahman, A. S. (2012), 'Genetic algorithm of sliding mode control design for manipulator robot', TELKOMNIKA (Telecommunication Computing Electronics and Control) 10(4), 645-654.

Firdaus, A. R. and Tokhi, M. (2015), Robust sliding mode-based interval type-2 fuzzy logic observer for quadcopter uavs, in 'System Theory, Control and Computing (ICSTCC), 2015 19th International Conference on', IEEE, pp. 559-564.

Firdaus, A. R. and Tokhi, M. (2016), Sliding mode control-based interval type-2 fuzzy logic controller for quadcopter uavs, in 'ASSISTIVE ROBOTICS: Proceedings of the 18th International Conference on CLAWAR 2015', World Scientific, pp. 555-563.

Ghabi, J. (2018), 'A novel sliding mode controller scheme for a class of nonlinear uncertain systems', International Journal of Modelling, Identification and Control 29(2).

Gonzalez-Hernandez, I., Palacios, F. M., Cruz, S. S., Quesada, E. S. E. and Leal, R. L. (2017), 'Real-time altitude control for a quadrotor helicopter using a super-twisting controller based on high-order sliding mode observer', International Journal of Advanced Robotic Systems 14(1), 1729881416687113.

Guo, M., Su, Y. and Gu, D. (2017), 'System identification of the quadrotor with inner loop stabilisation system', International Journal of Modelling, Identification and Control l 28(3).

Ibarra, E. and Castillo, P. (2017), Nonlinear super twisting algorithm for uav attitude stabilization, in 'Unmanned Aircraft Systems (ICUAS), 2017 International Conference on', IEEE, pp. 640-645.

Jasim, W. and Gu, D. (2019), 'Iterative linear quadratic regulator control for quadrotors leaderfollower formation flight', International Journal of Modelling, Identification and Control 31(2).

Liu, H., Derawi, D., Kim, J. and Zhong, Y. (2013), 'Robust optimal attitude control of hexarotor robotic vehicles', Nonlinear Dynamics 74(4), 1155-1168.
Liu, J. and Wang, X. (2011), Dynamic Sliding Mode Control, Springer Berlin Heidelberg, Berlin, Heidelberg, pp. 111-116.

Salgado, I., Chairez, I., Moreno, J., Fridman, L. and Poznyak, A. (2011), 'Generalized super-twisting observer for nonlinear systems', IFAC Proceedings Volumes 44(1), 14353 - 14358. 18th IFAC World Congress.

Schröder, D., Lenz, U., Beuschel, M., Hangl, F., Frenz, T., Strobl, D., Straub, S., Fischle, K., Rau, M. and Angermann, A. (2000), Intelligent Observer and Control Design for Nonlinear Systems, SpringerVerlag, Berlin Heidelberg.

Sebesta, K. D. and Boizot, N. (2014), 'A real-time adaptive high-gain ekf, applied to a quadcopter inertial navigation system', IEEE Transactions on Industrial Electronics 61(1), 495-503.

Shtessel, Y., Edwards, C., Fridman, L. and Levant, A. (2010), Sliding Mode Control and Observation, Springer-Verlag, New York.

Skender, M. R., Tlemçani, A. and Nouri, H. (2017), 'A novel observer algorithm of voltages across capacitors based on the higher sliding mode control: application to multi-cells converter', International Journal of Modelling, Identification and Control 27(2).

Slotine, J.-J., Hedrick, J. and Misawa, E. (1986), 'On sliding observers for nonlinear systems', Journal of Dynamic Systems, Measurement, and Control $\mathbf{1 0 9}(3), 245-252$.

Visioli, A. (2006), Practical PID Controll, SpringerVerlag, London.

Zanelli, A., Domahidi, A., Jerez, J. and Morari, M. (2017), 'Forces nlp: an efficient implementation of interior-point methods for multistage nonlinear nonconvex programs', International Journal of Control 0(0), 1-17.

Zheng, E.-H., Xiong, J.-J. and Luo, J.-L. (2014), 'Second order sliding mode control for a quadrotor uav', ISA transactions 53(4), 1350-1356. 